\title{
Stabilized equal low-order finite elements in ice sheet modeling - accuracy and robustness
}

\author{
Christian Helanow ${ }^{1}$ (D) . Josefin Ahlkrona ${ }^{2,3}$
}

Received: 6 March 2017 / Accepted: 15 December 2017 / Published online: 29 January 2018

(C) The Author(s) 2018. This article is an open access publication

\begin{abstract}
We investigate the accuracy and robustness of one of the most common methods used in glaciology for finite element discretization of the $\mathfrak{p}$-Stokes equations: linear equal order finite elements with Galerkin least-squares (GLS) stabilization on anisotropic meshes. Furthermore, we compare the results to other stabilized methods. We find that the vertical velocity component is more sensitive to the choice of GLS stabilization parameter than horizontal velocity. Additionally, the accuracy of the vertical velocity component is especially important since errors in this component can cause ice surface instabilities and propagate into future ice volume predictions. If the element cell size is set to the minimum edge length and the stabilization parameter is allowed to vary non-linearly with viscosity, the GLS stabilization parameter found in literature is a good choice on simple domains. However, near ice margins the standard parameter choice may result in significant oscillations in the vertical component of the surface velocity. For these reasons, other stabilization techniques, in particular the interior penalty method, result in better accuracy and are less sensitive to the choice of stabilization parameter. During this work, we also discovered that the manufactured solutions often used to evaluate errors in glaciology are not reliable due to high artificial surface forces at singularities. We perform our numerical experiments in both FEniCS and Elmer/Ice.
\end{abstract}

Keywords Finite element method · Galerkin least-squares $\cdot$ p-Stokes $\cdot$ Ice-sheet modeling $\cdot$ Anisotropic mesh

Mathematics Subject Classification $201076 \mathrm{M} 10 \cdot 86 \mathrm{~A} 40 \cdot 76 \mathrm{~A} 05 \cdot 76 \mathrm{~A} 20$

\section{Introduction}

Ice sheets and glaciers are important components of the climate system. Their melting is one of the main sources of sea-level rise [26], and in the past, major climatic events have been triggered by the dynamics of ice sheets [3, 37]. Numerical modeling is a key tool to understand both past and future evolution of ice sheets.

Christian Helanow

christian.helanow@natgeo.su.se

Josefin Ahlkrona

ahlkrona@math.uni-kiel.de

1 Department of Physical Geography, Stockholm University, Svante Arrhenius väg 8,106 91 Stockholm, Sweden

2 Division of Scientific Computing, Department of Information Technology, Uppsala University, Box 337, SE-751 05, Uppsala, Sweden

3 The Mathematical Seminar, University of Kiel, Westring 383, 24118 Kiel, Germany
The dynamics of ice sheets can be described as a very viscous, incompressible, non-Newtonian free surface, thinfilm flow, driven by gravity. The velocity field and pressure are given by the solution of a non-linear (steady-state) Stokes system $-\mathfrak{p}$-Stokes system. The position of the ice-atmosphere interface is computed by solving an additional convection equation where the velocity field enters as coefficients.

Early ice-sheet models used simple approximations of the governing equations discretized by the finite difference method [24, 36, 41]. In these models, some stress components are neglected, so that they are inaccurate in, e.g., the dynamical coastal regions but have the benefit of being computationally cheap. Since then, computational power has increased, and an accurate modeling of coastal regions has been identified as key to estimate future evolution of ice sheets. Consequently, some modern ice-sheet models now use the finite element method (FEM) to discretize the (nonapproximated), $\mathfrak{p}$-Stokes equations [22, 32, 44, 51, 60]. Compared to the early simplified models, more care has to 
be taken when choosing the discretization methods, as the $\mathfrak{p}$-Stokes equations comprise a computationally demanding, non-linear, saddle point problem. Numerical instabilities tend to occur at the so important coastal regions, and due to their computational expense, and in some cases instability, these models are limited to a few centuries if applied to the Greenland ice sheet, and an even shorter period if applied to the Antarctic ice sheet.

One of the most common choices of discretization is (bi/tri-) linear elements for both velocity and pressure. Linear equal order elements are appealing for three reasons. Firstly, they are easy to implement. This is important in large codes for geophysical simulations, as they often have many users/ developers from various research fields and include a large number of extra features and modules. Secondly, the regularity of the solutions to p-Stokes solutions is limited [12, 38], so that the usage of higher order elements might not result in a higher order of convergence. Thirdly, they might be more efficient than higher order elements, which results in denser matrices. This is especially true on supercomputers and for non-linear applications [16, 34]. Furthermore, ice-sheet modeling usually involves data with high uncertainty so that a elements may outperform higher order elements for any realistic accuracy requirement. It is well-known, however, that equal order linear elements do not satisfy the so-called inf-sup condition [7, 12, 20, 21], which leads to instabilities. To circumvent this, stabilization techniques are introduced.

A-priori analysis for the $\mathfrak{p}$-Stokes equations discretized with inf-sup stable isotropic elements was done in, e.g., [12, 43], and for d-linear equal order isotropic elements stabilized with a local projection stabilization in [38]. The accuracy, robustness, and stability of equal order stabilized methods for practical ice simulations are however not well established. Ice-sheet simulations involve the solution of the $\mathfrak{p}$-Stokes equations on very flat domains (i.e., on anisotropic meshes), with complex boundaries and boundary conditions. In a recent review study, anisotropic meshes and realistic boundary conditions for incompressible flow problems, were identified as two of the most important at large open problems of finite element research [42], without overlooking important works of, e.g., [2, 48]. The authors also called for more systematic studies or stabilized methods and choices of stablization parameters for practical simulations. A rigorous analytical understanding of the accuracy and robustness of practical ice-sheet simulations is thus probably out of reach within the near future, at the same time as accurate and robust simulations are urgent, given their importance for understanding future sea-level rise and climate.

In this study, we therefore perform numerical experiments to assess the numerical errors, convergence, and robustness of equal order linear elements with one of the most common stabilizations used ice-sheet modeling: the Galerkin least-squares (GLS) stabilization [31]. We also investigate how the errors influence the accuracy of ice surface position calculations and how they depend on the GLS stabilization parameter, as it is currently not clear how numerical errors resulting from solving the discretized $\mathfrak{p}$-Stokes system interplay with the simulations of the ice surface evolution and, ultimately, predictions of future ice volume. Finally, we test and compare with alternative stabilization methods, namely a pressure penalty method, an interior penalty method [23], a pressure global projection method [27], and the local projection stabilization [11], some of which overcome issues found with the GLSstabilized method. We hope that this study will spark more future studies, assessing the numerical stability and accuracy of ice-sheet models.

The experiments are initially performed with two different finite element codes that have been used with success in glaciology: FEniCS [4, 47], which is the underlying framework of the ice sheet model VarGlaS [22], and Elmer/Ice [32], which is built on the Elmer software [52]. FEniCS uses tetrahedra (triangles in 2D), while Elmer/Ice commonly uses prismatic or quadrilateral elements (triangles or rectangles in 2D). Implementing the same problems in two different codes has helped to demonstrate problems related to the underlying equations and identify any issues related to specific implementations. In doing so, we hope that the insights of this paper is applicable to a wide range of icesheet codes. Once we established that the two codes behave similarly, we make further experiments in FEniCS.

We also use two different methods for measuring accuracy, namely by using manufactured solutions [45], and by a reference solution. Manufactured solutions has gained some popularity as a model validation method in glaciology. We use both methods because we have observed some issues with the usage of the manufactured solutions, while a numerical reference solution is feasible only in $2 \mathrm{D}$ due to computational cost. By using both methods for some of our experiments, we can differentiate between issues related to the manufactured problem and errors arising from the numerical method. It also allows us to discuss if and when manufactured solutions are useful.

The paper is structured as follows. The governing equations and the standard equal order GLS-stabilized finite element method are described in Section 2, together with the FEM codes used. In Section 3, the accuracy and convergence of the equal order GLS stabilization is measured using both FEniCS and Elmer/Ice using standard parameter settings. This is done both with manufactured and reference solutions for the ISMIP-HOM set-up. In Section 4, we study how the numerical errors measured in Section 3 propagate to the evolution of the free ice surface. The sensitivity of the accuracy to the GLS stabilization parameter is measured in 
Section 5, while Section 6 treats how the stability parameter depends on viscosity and finite element cell size. Finally, alternative stabilization techniques are tested in Section 7 , with regards to accuracy and parameter sensitivity. Our main conclusions are presented in Section 8.

\section{Theory and background}

\subsection{Mathematical model}

\subsubsection{Governing equations}

We consider an ice sheet or glacier domain $\Omega$ in a Cartesian coordinate system $(x, y, z)$, see Fig. 1 . Note that the sketch in Fig. 1 is exaggerated in the vertical direction, and that in reality, ice sheets are very thin.

The ice velocity $u=\left(u_{x}, u_{y}, u_{z}\right)$ and pressure $p$ are given by the solution to the $\mathfrak{p}$-Stokes equations

$$
\begin{array}{r}
-\nabla p+\nabla \cdot \mathrm{S}+\rho g=0 \\
\nabla \cdot \mathrm{u}=0
\end{array}
$$

where $\rho$ is the ice density, $\rho g$ the gravitational force, and $S$ is the deviatoric stress tensor. The deviatoric stress tensor is related to the strain rate tensor $\mathrm{D}=\frac{1}{2}\left(\nabla \mathrm{u}+\nabla \mathrm{u}^{T}\right)$ through a power law,

$$
\begin{aligned}
\mathrm{S}(\mathrm{u}) & =2 \eta(|\mathrm{D}|) \mathrm{D}, \\
\eta & =\eta_{0}|\mathrm{D}|^{\mathfrak{p}-2},
\end{aligned}
$$

where $|\mathrm{D}|^{2}=\mathrm{D}$ : $\mathrm{D}$ is the second invariant of the strain rate tensor. The power-law parameter $\mathfrak{p}$ indicates the nonlinearity of the material and $\eta_{0}$ is a large temperaturedependent parameter. In this study, we consider the isothermal case to focus on the effects resulting from the discretization of Eq. 1. In glaciology, Eq. 2b is called Glen's flow law and $\mathfrak{p}=1 / n+1$, where $n$ is the so-called Glen's parameter. We will apply the standard value $n=3$, so that $\mathfrak{p}=4 / 3$. Since $\mathfrak{p}<2$ ice is a shear thinning fluid and has infinite viscosity as the strain rate tensor approaches zero.

Inserting Eq. 2 into Eq. 1a yields

$-\nabla p+\nabla \cdot\left(\eta(|D|)\left(\nabla u+(\nabla u)^{T}\right)\right)+\rho g=0$

The ice surface, $s$, deforms according to the velocity field, $\left.u\right|_{z=s}$, and its evolution given by free surface equation

$\partial_{t} s+u_{x} \partial_{x} s+u_{y} \partial_{y} s-u_{z}=a_{s} \quad$ for $z=s$

The net accumulation of the ice sheet, $a_{s}$, depends on precipitation and surface air temperature. The free surface problem Eq. 4 is solved only on the ice surface.

\subsubsection{Boundary conditions}

The boundary of the glacier domain, $\partial \Omega$, consists of the glacier bed, the surface, and the horizontal boundaries, $\partial \Omega=\Gamma_{s} \cup \Gamma_{h} \cup \Gamma_{f} \cup \Gamma_{\beta^{2}}$. At the ice surface, $\Gamma_{s}$, and horizontal boundaries, $\Gamma_{h}$, wind stresses and atmospheric pressure are neglected so that the stress-free condition holds.

At the ice-bedrock interface, $\Gamma_{f} \cup \Gamma_{\beta^{2}}$, the conditions depend on, e.g., thermal and hydrological conditions. We consider grounded ice sheets that are either frozen to the bed (so that a no-slip condition applies) or that slide against the bed. Together we have that

$$
\begin{array}{cl}
\eta(|D|)\left(\nabla u+(\nabla u)^{T}\right) \cdot n-p n=0, & \\
& \text { on } \Gamma_{s} \cup \Gamma_{h}, \\
u=0, & \text { on } \Gamma_{f}, \\
\left(\eta(|D|)\left(\nabla u+(\nabla u)^{T}\right) \cdot\right. & n-p n)^{\|}=-\beta^{2} u^{\|}, \\
& \text {on } \Gamma_{\beta^{2}}, \\
u \cdot n=0, & \text { on } \Gamma_{\beta^{2}},
\end{array}
$$

where $\beta^{2}>0$ is the coefficient of basal traction, $\Gamma_{f}$ and $\Gamma_{\beta^{2}}$ denote the parts of the bed where the conditions are frozen

Fig. 1 A body of ice in a Cartesian coordinate system.

The ice surface $s=s(x, y, t)$ evolves in time, while the base $b=b(x, y)$ is considered rigid. The surface normal vector, $n$ is pointing outwards from the ice. In two dimensions there is one tangential vector $t$, and in three dimensions two orthogonal vectors $t_{1}$ and $t_{2}$ span a tangential plane. Black arrows indicates velocity patterns, $u(x, y, z, t)$

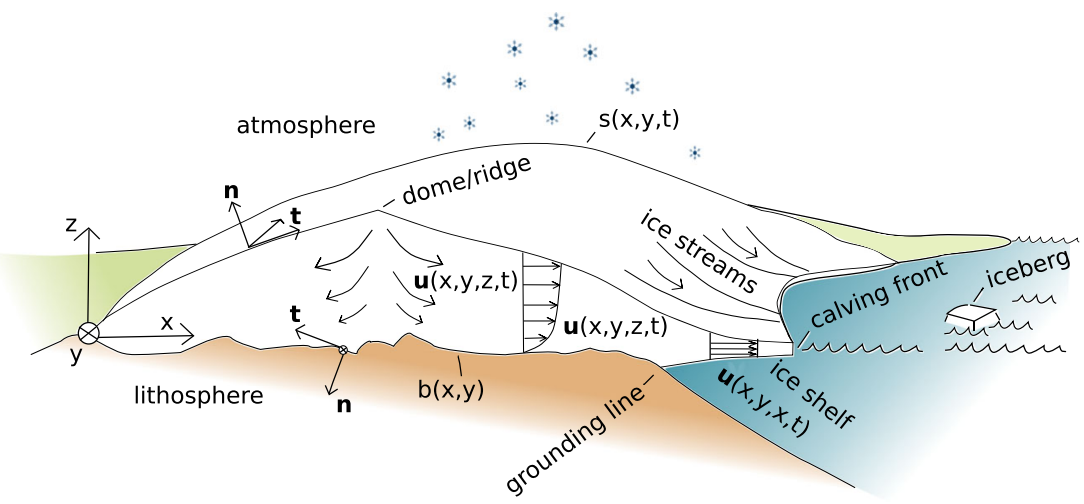


and sliding, respectively, and $\|$ denotes the components of a vector that are tangential to the bed. The well-posedness of the system with various relevant boundary conditions relevant for glaciology has been studied in $[25,43]$.

\subsection{Discretization methods}

\subsubsection{Overall solution strategy}

The standard approach to solve for the velocity field, pressure, and the evolution of the ice surface position is outlined in Algorithm 1. For brevity of presentation, the non-linearity is resolved with a Picard iteration in Algorithm 1. However, this may be exchanged for a more efficient Newton iteration, if correct precautions are taken to ensure robustness.

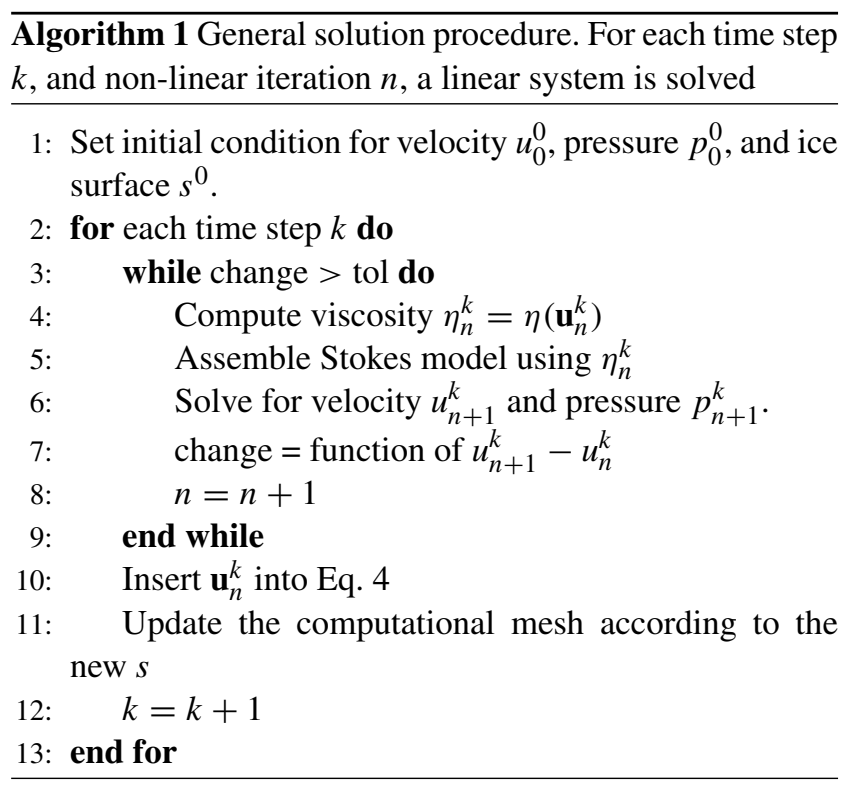

For zero strain rates, the linear system assembled in Algorithm 1 is ill-conditioned, and in case of a Newton iteration, the Jacobian matrix does not exist [38]. The standard method to avoid this is to introduce a small parameter, the critical shear rate, to the constitutive law Eq. $2 \mathrm{~b}$, so that infinite viscosity is avoided at zero strain. We choose the critical shear rate as small as possible for convergence, so that it does not significantly influence our results (see detailed information in Sections 2.2.3 and 2.2.4).

\subsubsection{Finite element formulation}

The weak formulation of the $\mathfrak{p}$-Stokes system in Eq. 1 reads:

Find $(u, p) \in V \times Q$ such that

$$
\begin{aligned}
A(u, v)+B(v, p) & =F(v) \quad \forall v \in V, \\
B(u, q) & =0 \quad \forall q \in Q,
\end{aligned}
$$

with

$$
\begin{aligned}
A(u, v) & =\int_{\Omega} S(u): \nabla v d \Omega \\
& =\int_{\Omega}\left(\eta(|D|)(\nabla u)+\nabla(u)^{T}\right): \nabla v d \Omega, \\
B(v, p) & =\int_{\Omega}-p \nabla \cdot v d \Omega, \\
F(v) & =\int_{\Omega} \rho g \cdot v d \Omega,
\end{aligned}
$$

where $V:=\left\{v \in\left[W^{1, \mathfrak{p}}(\Omega)\right]^{d}:\left.v\right|_{\Gamma_{f}}=0,\left.v \cdot n\right|_{\Gamma_{\beta^{2}}}=0\right\}$ and $Q:=L^{\mathfrak{p} /(\mathfrak{p}-1)}(\Omega), V \times Q$ being either $x-y$ periodic or stress-free on $\Gamma_{h}$. The associated discretized problem is:

Find $\left(u_{h}, p_{h}\right) \in V_{h} \times Q_{h}$ such that

$$
\begin{aligned}
A\left(u_{h}, v_{h}\right)+B\left(v_{h}, p_{h}\right) & =F\left(v_{h}\right) \quad \forall v_{h} \in V_{h} \\
B\left(u_{h}, q_{h}\right) & =0 \quad \forall q_{h} \in Q_{h},
\end{aligned}
$$

or equivalently,

Find $\left(u_{h}, p_{h}\right) \in V_{h} \times Q_{h}$ such that

$$
\begin{aligned}
& A\left(u_{h}, v_{h}\right)+B\left(v_{h}, p_{h}\right)+B\left(u_{h}, q_{h}\right) \\
& =F\left(v_{h}\right) \quad \forall\left(v_{h}, q_{h}\right) \in V_{h} \times Q_{h},
\end{aligned}
$$

where $V_{h}$ and $Q_{h}$ are finite element space restrictions of $V$ and $Q$, respectively, defined on a partition (mesh) $T_{h}$ of $\Omega$ with mesh size $h$. The meshes used in ice-sheet modeling are highly anisotropic, since the vertical extent of the ice sheet is much smaller than its lateral extent. Meshes are usually layered, following the topography. The number of layers in an ice-sheet simulation are usually 15-20. For an ice sheet that is a couple of kilometers thick the typical vertical edge size is thus about $200 \mathrm{~m}$ while the resolution in the horizontal plane can vary from $50 \mathrm{~km}$ down to $500 \mathrm{~m}$ if mesh adaptation is used [33, 54]. The spaces $V_{h}$ and $Q_{h}$ have to fulfill the inf-sup condition [7, 12, 20, 21]. If the inf-sup condition is violated, spurious pressure oscillations or locking of the velocity field may occur. An example of a choice that leads to a stable discretization is the so-called Taylor-Hood element [56] (stable on isotropic meshes), which consists of piecewise quadratic polynomials for the velocity and piece-wise linear for the pressure. Many ice-sheet models apply linear polynomial functions ( $P 1 P 1$ for tetrahedrons/triangles) or bi/tri-linear ( $Q 1 Q 1$ for prisms/quadrilaterals) for both the velocity and pressure space $[60,61]$. Equal order elements are preferred due to their simple implementation, the limited regularity of the $\mathfrak{p}$-Stokes equations and moderate accuracy requirements. Unfortunately, such equal order elements do not fulfill the inf-sup condition. To circumvent this issue, stabilization techniques are used. 
The GLS method $[31,40]$ is one of the most common stabilization techniques. In this method, extra terms $S_{G L S}$ and $F_{\mathrm{GLS}}$ are added to the discrete form as

$$
\begin{aligned}
& A\left(u_{h}, v_{h}\right)+B\left(v_{h}, p_{h}\right) \\
& +B\left(u_{h}, q_{h}\right)+S_{G L S}\left(\left(u_{h}, p_{h}\right),\left(v_{h}, q_{h}\right)\right)= \\
& F\left(v_{h}\right)+F_{G L S}\left(\left(v_{h}, q_{h}\right)\right), \quad \forall\left(v_{h}, q_{h}\right) \in V_{h} \times Q_{h} .
\end{aligned}
$$

The stabilization terms $S_{\mathrm{GLS}}$ and $F_{\mathrm{GLS}}$ are defined as

$$
\begin{aligned}
& S_{\mathrm{GLS}}\left(\left(u_{h}, p_{h}\right),\left(v_{h}, q_{h}\right)\right)= \\
& -\sum_{K \in T_{h}} \tau_{\mathrm{GLS}}\left(-\nabla \cdot S(u)+\nabla p_{h},-\nabla \cdot S(v)+\nabla q_{h}\right)_{K} \\
& F_{\mathrm{GLS}}\left(\left(v_{h}, q_{h}\right)\right)= \\
& -\sum_{K \in T_{h}} \tau_{\mathrm{GLS}}\left(\rho g,-\nabla \cdot S(v)+\nabla q_{h}\right)_{K}
\end{aligned}
$$

where $K$ denotes triangles/quadrilaterals or tetra-/hexahedrons in the partition $T_{h}$, and $(\cdot, \cdot)_{K}$ denotes the $L^{2}$ inner product in the element $K$. The GLS method is called a consistent method because the extra terms vanish for the true strong solution. However, for $P 1 P 1$ elements on triangles/tetrahedrons second derivatives vanish, leading to $\nabla \cdot S=0$. For prismatic or quadrilateral elements, a partial dependency on the gradient of the velocity in Eq. 10 remains through the bi/tri-linear term. This contribution is however small unless elements are highly distorted.

In [31] the stabilization parameter is

$\tau_{\mathrm{GLS}}=\tau_{0} \frac{m_{K} h_{K}^{2}}{8 \eta}$,

where $m_{K}=1 / 3$ for linear interpolations. The size of the stabilization parameter thus depends on how the element measure, i.e., the cell size $h_{K}$, is defined (this will be discussed in Section 6.1). For non-Newtonian fluids, $\tau_{\mathrm{GLS}}$ also varies with the viscosity $\eta$. The parameter $\tau_{0}$ does not occur in the original formulation in [31]. It is introduced in the present study to investigate the effects of over- and under-stabilization. Its default value is therefore $\tau_{0}=1$. In a simulation, over or under-stabilization might occur if the GLS formulation is not an suitable method for the particular problem, if the analysis in [31] is not valid for the problem, or if the parameters $h_{K}$ and $\eta$ are not chosen appropriately. In initial tests, we let $\tau_{0} \neq 1$ represent such a situation, and further on in the study, we investigate how $\tau_{0}$ can differ from one in order to find an accurate solution.

In the case of a linear Newtonian fluid, using GLSstabilized equal order linear elements, the error of the pressure and velocity gradient (measured in the $L^{2}$-norm) is expected to decrease linearly with the cell size $h$ (on isotropic meshes). This implies an $\mathcal{O}\left(h^{2}\right)$ convergence in velocity if the solution to the problem is smooth enough [30]. For the non-Newtonian p-Stokes case, analyses of how the pressure and velocity gradients converge are available for equal order (isotropic) elements with local projection stabilization [39] and for some (isotropic) inf-sup stable elements including the MINI element [12]. These studies suggest an $\mathcal{O}\left(h^{1}\right)$ convergence for the velocity gradient measured in the $L^{\mathfrak{p}}$ norm, and in $[12,39]$ an $\mathcal{O}\left(h^{2(\mathfrak{p}-1) / \mathfrak{p}}\right)$ convergence in pressure (i.e., $\mathcal{O}\left(h^{0.5}\right)$ for ice), measured in the $L^{\mathfrak{p} /(\mathfrak{p}-1)}$ norm. The convergence of the pressure is thereby lower than the $\mathcal{O}\left(h^{1}\right)$ interpolation error. This is due to the non-linear stress tensor. On simple enough problems however, where the regularity is high enough, an $\mathcal{O}\left(h^{1}\right)$ pressure convergence can be observed. The study of [12] is interesting since there is a link between GLS methods and the MINI element (i.e., bubbles stabilization) [8]. However, it has been shown in [58] that equivalence between the two methods does not hold with standard bubble functions for Stokes flow on hexahedral or quadrilateral elements, so that the relation is unclear for ice-sheet modeling.

\subsubsection{Implementation and mesh generation in FEniCS}

FEniCS is a set of fully parallelized software components for solving partial differential equations by the finite element method. The core is written in $\mathrm{C}^{++}$and also has a Python-interface. Notably, one of the high-level features is the use of a (Python like) form language (UFL, [5]) which closely resembles the mathematical language used for weak formulations of partial differential equations. FEniCS supports a wide variety of finite elements defined on triangles or tetrahedrons.

In this study, the $P 1 P 1$ element was used. We used the built-in mesh generation to deform a structured mesh on a cuboid (in 3D, consisting of tetrahedrons) or a rectangular (2D, triangles) to the desired surface and bed geometry.

The non-linearity was solved by a manually programmed Newton method in which, for each iteration, the linear subproblem was solved with a direct linear solver from the PETSc [9] package.

In the FEniCS implementation of this study, a critical shear rate which regularizes the material singularity is added to Eq. $2 \mathrm{~b}$ so that $\eta=\eta_{0}|D+\dot{\gamma}|^{\mathfrak{p}-2}$. The value is chosen as as $\dot{\gamma}=1 e^{-14}$ (corresponding to velocities measured in $\mathrm{m} y r^{-1}$ ), which is the smallest possible that allows convergence when singular values of the effective viscosity are present.

\subsubsection{Implementation and mesh generation in Elmer/Ice}

Elmer/Ice is based on the multiphysics code Elmer [32]. It is written in Fortran 90, and has been used to perform large scale, parallelized ice-sheet simulations, e.g., [33, 54].

So far, equal order linear elements $(Q 1 Q 1)$ are used for ice-sheet simulations with Elmer/Ice. The standard approach to construct a $3 \mathrm{D}$ mesh is to first create a $2 \mathrm{D}$ footprint mesh consisting of triangles or quadrilaterals and 
extrude this in the vertical into a 3D mesh consisting of prismatic or hexagonal mesh, see Fig. 2. In this study, hexagonal meshes are used in the Elmer/Ice simulations.

As the ice surface evolves, the extruded mesh allows for shifting nodes only in the vertical direction, which is computationally efficient.

Unlike FEniCS, the weak forms and stabilization methods are already implemented, and only parameter choices are easily adjustable. Available stabilization techniques are GLS stabilization (as will be used in this study), or the MINI-element (residual free bubbles method) [6, 8]. The residual free bubbles method is computationally costly due to the extra degrees of freedom.

The non-linearity in Algorithm 1 was solved by a Picard iteration (even though a Newton iteration is available), in which for each iteration, the linear sub-problem was solved with a direct linear solver from the UMFPACK package [28].

In Elmer/Ice, the critical shear rate $\dot{\gamma}$ is not added to the effective strain rate, as in FEniCS, but is instead used as a lower threshold of $|D|$. The value of $\dot{\gamma}$ is $1 e^{-10}$ (corresponding to velocities measured in $\mathrm{m} \mathrm{yr}^{-1}$ ).

\section{Accuracy, convergence, and evaluating measuring techniques}

In this section, we assess how the numerical errors or the finite element discretization of the $\mathfrak{p}$-Stokes equations decrease with the cell size, how large the errors are, and how they are distributed. This is done for the default stabilization parameter (i.e., $\tau_{0}=1$ ) on the benchmark set-up ISMIP-HOM A and $\mathrm{B}[50]$.

\subsection{Experiment set-up}

The ISMIP-HOM A experiment describes ice flow over an infinite, inclined sinusoidal bed (see Fig. 3) with no slip conditions at the base. The bed, $b$, and surface, $s$, are

$$
\begin{aligned}
& s(x, y)=-\tan (\alpha) x \\
& b(x, y)=s(x, y)-Z+\frac{Z}{2}\left[\sin \left(\frac{2 \pi x}{L}\right) \sin \left(\frac{2 \pi y}{L}\right)\right],
\end{aligned}
$$

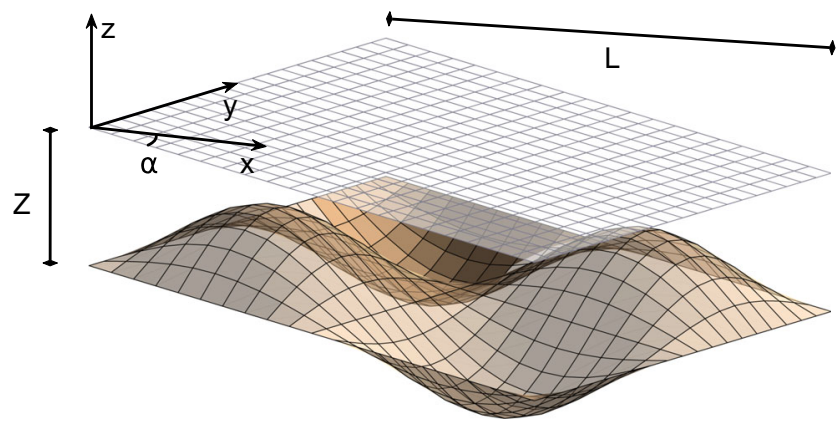

Fig. 3 The ISMIP-HOM A domain. The ice is $Z=1 \mathrm{~km}$ thick on average and is flowing down a plane of inclination $\alpha=0.5^{\circ}$ and length $L=80 \mathrm{~km}$. The bed has sinusodial bumps of amplitude $Z / 2=500 \mathrm{~m}$

where $Z=1 \mathrm{~km}$ is the typical thickness of the ice, $L=$ $80 \mathrm{~km}$ is the horizontal ( $x$ and $y$ ) extension of the domain and $\alpha=0.5^{\circ}$ is the inclination of the surface. For the ISMIP-HOM B experiment, the $y$-dependency is excluded. The ISMIP-HOM benchmark is described in more detail in [50].

Measuring accuracy and convergence is non-trivial on a non-linear problem with singularities. No analytical solution exists for the ISMIP-HOM problem, and obtaining a numerical reference solution for a three-dimensional problem is computationally demanding. Therefore, $[45,53]$ developed a manufactured solution which provides an analytical reference solution to a problem with altered surface boundary conditions and body force. These solutions have been used to measure convergence rate and accuracy for the ISMIP-HOM benchmark experiment A in, e.g., [22, 32, 45] (slightly altered).

As the manufactured solutions introduce artificial surface and body forces, there is a risk that the accuracy observed for the manufactured problem does not apply for the original problem. To our knowledge, the quality of the manufactured solutions of [45] has not been studied. Therefore, we also use a numerical reference solution for ISMIP-HOM B experiment, the two-dimensional version of the ISMIPHOM A experiment. This reference solution is obtained by solving the problem on a very fine mesh. It would of course be optimal to have a numerical reference solution for the three-dimensional ISMIP-HOM A experiment, but this was too computationally demanding. To generate the
Fig. 2 Extruded mesh for the Greenland ice sheet

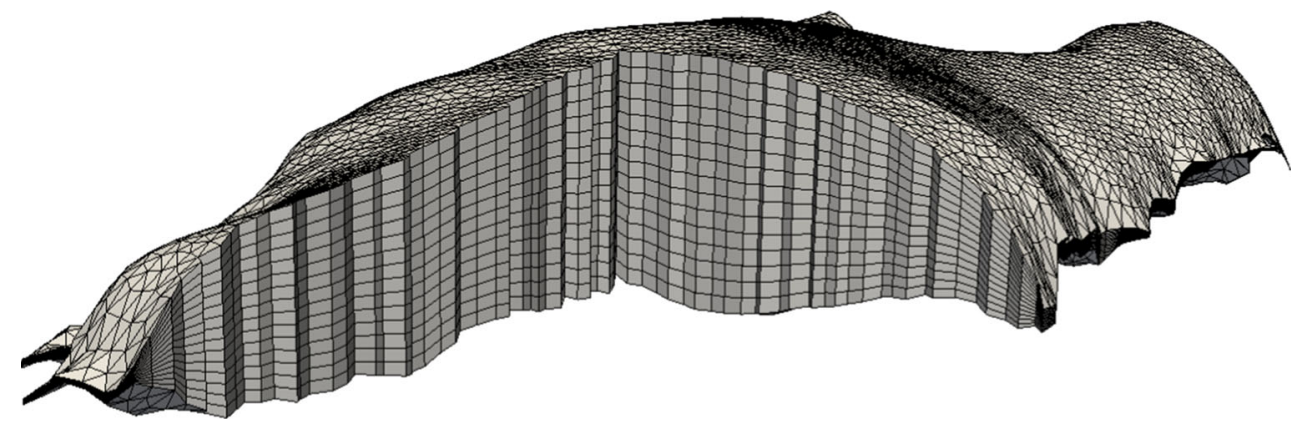


manufactured solution and artificial boundary conditions and forcing, a code modified from the supplementary material of [45] was used in both FEniCS and Elmer/Ice.

We employ 20 different meshes, the reference solution excluded. These meshes include five different resolutions in the horizontal direction, $N_{X}=10,20,40,80,160$ and four different resolutions in the vertical direction, $N_{Z}=5,10$, 20, 40, where $N_{X}$ and $N_{Z}$ denotes the number of elements in respective direction. Throughout this paper, the mesh with $N_{X}=20$ and $N_{Z}=20$ will be regarded as the resolution closest to those used in practical ice sheet simulations, i.e., a horizontal resolution of $4 \mathrm{~km}$ and a vertical resolution of about $50 \mathrm{~m}$. The numerical reference solution is computed for $N_{X}=1280$ and $N_{Z}=640$ with FEniCS, whereas a coarser resolution of $320 \times 80$ is used in Elmer/Ice. The simulations were performed with $h_{K}$ defined as the minimum edge length.

We choose to follow the approach in earlier studies, e.g., $[32,45]$, and present the errors in the $L^{2}$-norm instead of

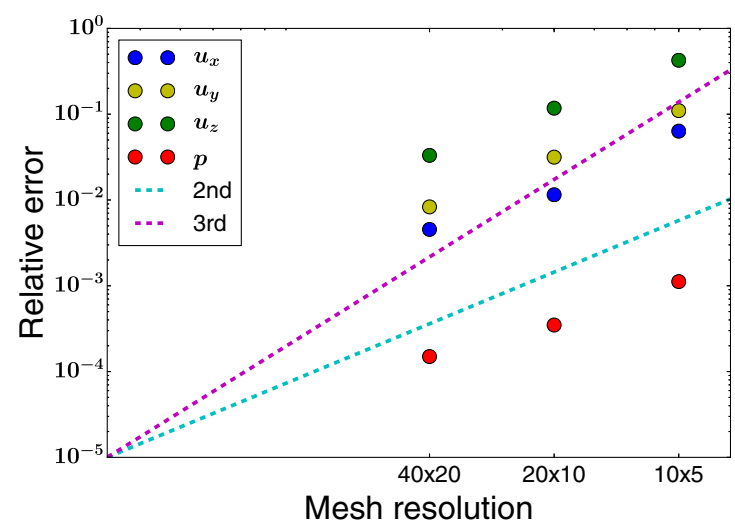

(a) Accuracy compared to a manufactured solution, FEniCS (P1P1)

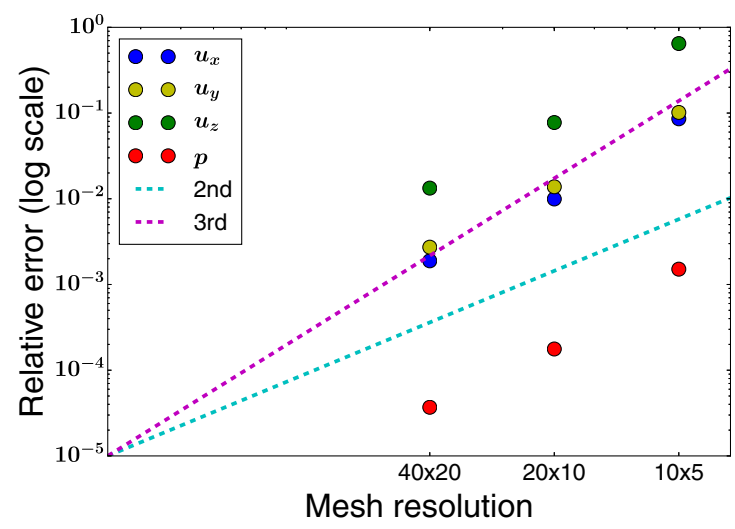

(c) Accuracy compared to a manufactured solution, Elmer/Ice (Q1Q1)

Fig. 4 Convergence of velocity components and pressure for equal order (bi/tri-)linear elements with standard GLS stabilization, computed by FEniCS ( $P 1 P 1$ element) and Elmer/Ice $(Q 1 Q 1$ element). The accuracy was measured in comparison to both a manufactured the for the $\mathfrak{p}$-Stokes problem more natural spaces $L^{\mathfrak{p} /(\mathfrak{p}-1)}$ and $W^{1, \mathfrak{p}}$. In future studies it would be interesting to also include the $\mathrm{H}^{1}$-norm, as this may be more sensitive to overand understabilization.

\subsection{Results}

\subsubsection{Convergence}

The relative error in velocity components and pressure for varying grid resolutions is shown in Fig. 4 for FEniCS ( $P 1 P 1$ element) and Elmer/Ice ( $Q 1 Q 1$ element), using both a manufactured solution and a numerical reference solution.

Both FEniCS and Elmer/Ice show second-order convergence in the velocity when compared to a numerical reference solution. In Elmer, the convergence rate is also second order for the pressure. This is higher than expected even for the linear Stokes problem. Such super-approximation

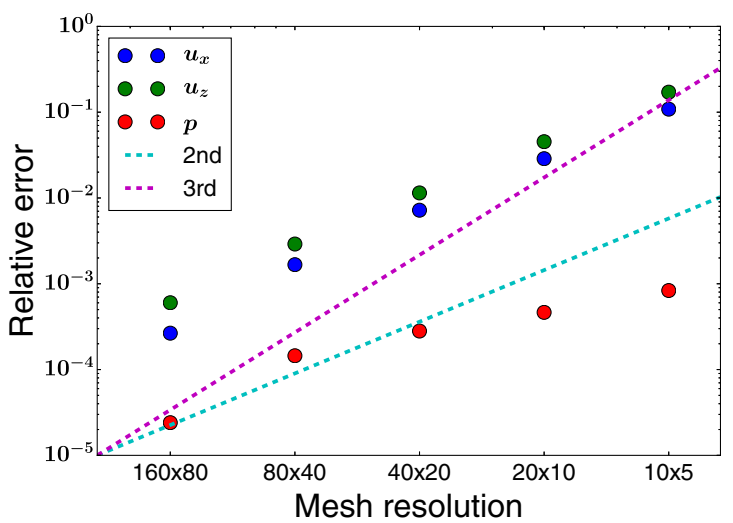

(b) Accuracy compared to a numercal reference solution, FEniCS $(P 1 P 1)$

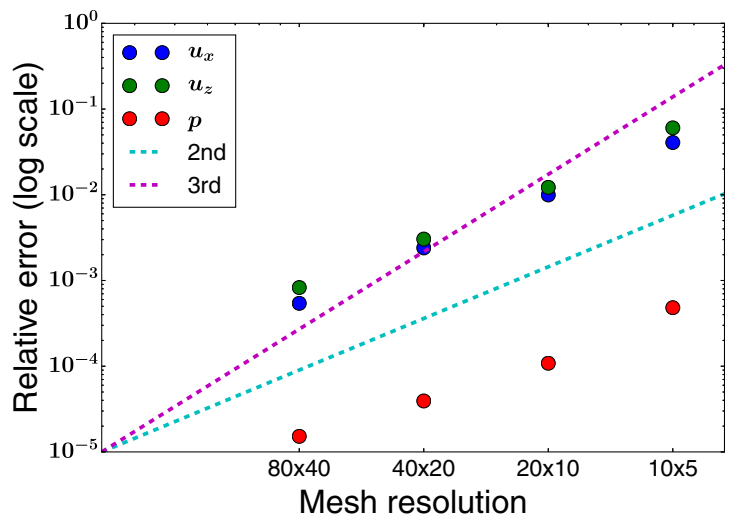

(d) Accuracy compared to a numerical refernce solution, Elmer/Ice $(Q 1 Q 1)$

solution (panel a, c) and for the original problem using a numerical reference solution (panel b, d). Dashed lines indicate second (cyan) and third (magenta) order convergence rates 
can occur for smooth enough solutions [30] and was also observed for the $\mathfrak{p}$-Stokes problem in [38]. When compared to the manufactured solution, the rate of convergence in FEniCS is two, while in Elmer/Ice it increased to three. The unexpected third-order convergence was obtained also in [32] for the manufactured problem, not only with linear elements with GLS stabilization, but also with residual free bubbles stabilization and higher-order elements. When comparing Elmer/Ice to the manufactured solutions, the convergence rate is two under horizontal refinement, and almost four under vertical refinement. Refining in both the horizontal and vertical direction combines to the thirdorder convergence. The higher convergence order under vertical refinement is seen also in FEniCS, where it is three instead of Elmer/Ice's four, but the combined horizontal and vertical refinement results in the expected second-order convergence rate. For a linear viscosity law, the convergence rate is two even with Elmer/Ice on the manufactured solutions, and the and numerical errors are overall lower.

A probable explanation of the unexpected results is that for the manufactured problem, the introduced artificial surface stresses and body force are functions of the (calculated) viscosity and may therefore approach infinity in areas where the effective shear rate approaches zero. This is of course not viable in the discrete case and an upper limit is set for the viscosity to keep the terms from getting too large. However, even with this limit, the artificial stresses become very high and the convergence to the solution is poor. To obtain convergence, we follow $[32,45]$ and set the artificial surface stress to zero at these manufactured singular points. However, we believe that this is a somewhat unphysical solution to the problem, and that the high artificial stresses can affect the problem to a degree where it neither is representative for ice sheet scenarios nor a problem for which it is possible to get a representative convergence rate. The errors and convergence is sensitive to implementation details and it is likely that the high-compensatory terms in the vicinity of the singularity in the manufactured problem is the cause of the unexpected third-order convergence of Elmer/Ice. Note that the above discussion does not exclude the possibility that some of these effects may be due to that the manufactured solutions are for the 3D ISMIP-HOM A problem and the numerical reference solution is for the $2 \mathrm{D}$ ISMIP-HOM B problem. To be certain, we did also perform the simulations using the manufactured simulations in $2 \mathrm{D}$, with parameters from [53], which qualitatively gave the same results.

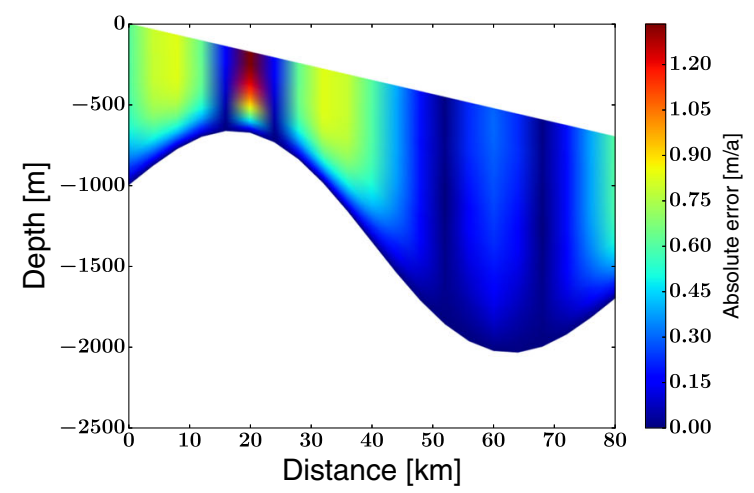

(a) Error of horizontal velocity, $u_{x}$

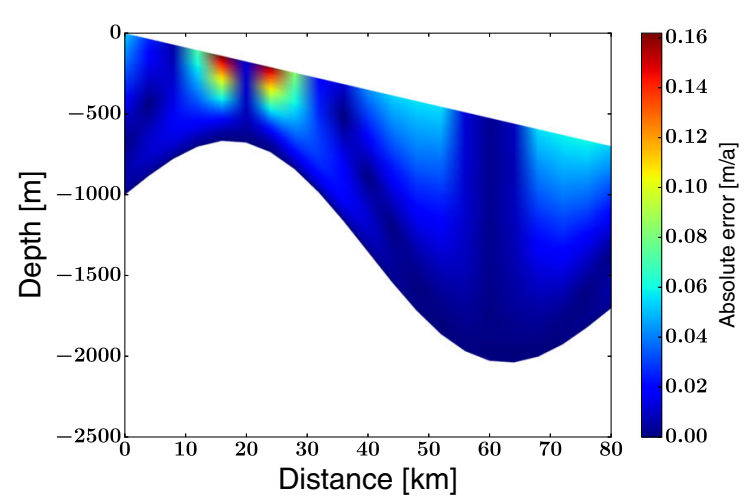

(c) Error of vertical velocity, $u_{z}$

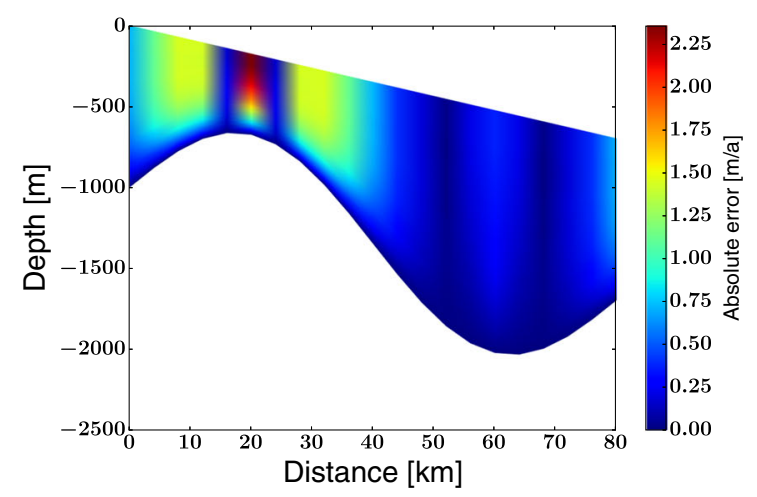

(b) Error of horizontal velocity, $u_{y}$

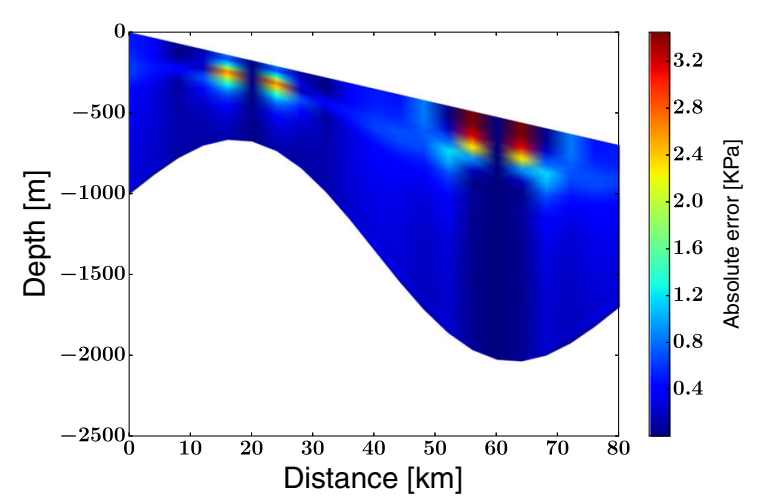

(d) Error of pressure, $p$

Fig. 5 Absolute error of Elmer/Ice in comparison for the manufactured problem on a $20 \times 20 \times 20$ grid, shown along the cross-section $y=L / 4$ 


\subsubsection{Error distribution}

The absolute errors for the velocity and pressure fields on the manufactured problem are shown in Fig. 5 (along crosssection $y=L / 4)$. The errors relative to the numerical reference are shown in Fig. 6. Since the results are similar for FEniCS and Elmer/Ice in these simulations (i.e., using $P 1 P 1$ and $Q 1 Q 1$ elements show the same characteristics), we here only show the Elmer/Ice output. The errors at $x=$ $L / 4$ and $x=3 L / 4$ are due to the non-linear viscosity, and for a linear case only the high errors at $x=0, x=L / 2$, and $x=L$ remains. These errors are expected to increase if the wave-length of the bedrock-variation decreases, since the wave-length $L$ is related to the non-linearity of the problem.

Both the magnitude and distribution is very different for the manufactured case in Fig. 5 compared to the original case in Fig. 6, especially so at $x=L / 4$ and $x=3 L / 4$ where singularities occur and the viscosity is infinite in the continuous case. Another difference between using a numerical reference solution and manufactured solution is that for the latter, the vertical velocity error is significantly higher than the horizontal velocity error, both in FEniCS and Elmer/Ice . This effect is not as prominent when the numerical reference is used.

Since neither convergence rates, nor error magnitude and distribution is well represented using the manufactured solutions, we will in the remaining experiments of this paper study the original problem using the numerical reference solution. Only in Section 5.1 will we use the manufactured problem again to ensure that the stability term in the numerical reference solution does not bias certain results. Note that the manufactured solutions can be useful in some cases, as in the mass conservation studies of [46].

\section{Propagation of errors to the free surface equation}

In this section, we investigate how the errors in the velocity propagate to the solution of the ice surface position, which they do since the velocity components at the ice surface enter as coefficients in the free surface evolution equation Eq. 4. As can be seen from Fig. 6, these errors are highest near the surface which directly affects the free surface.

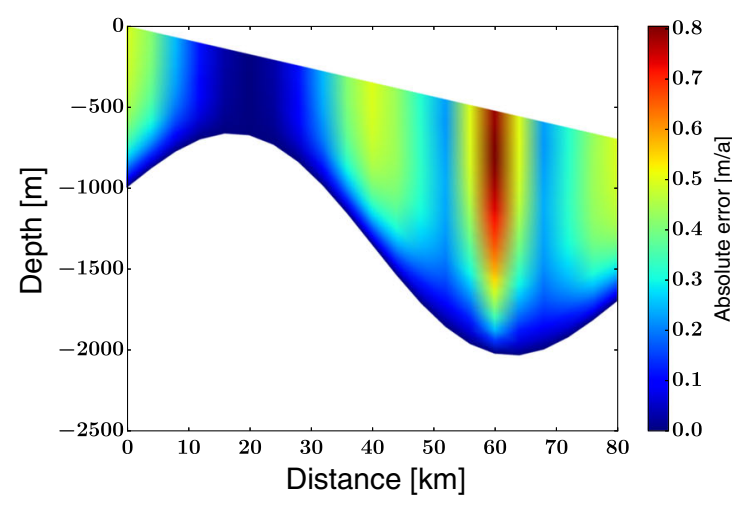

(a) Error of horizontal velocity, $u_{x}$

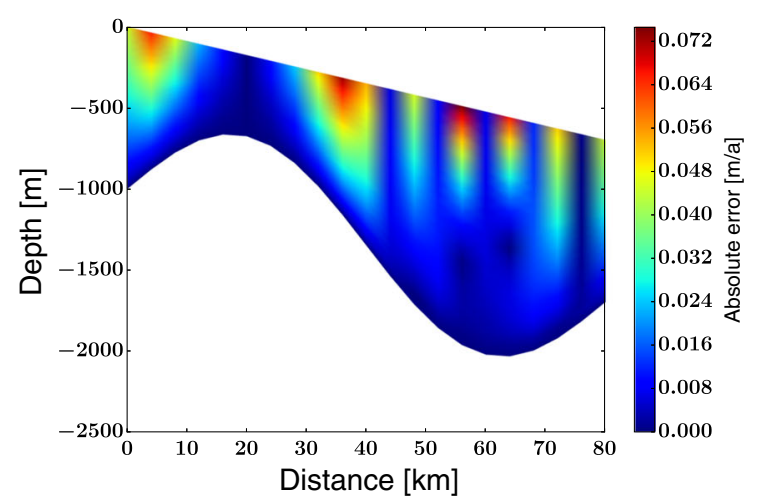

(b) Error of vertical velocity, $u_{z}$

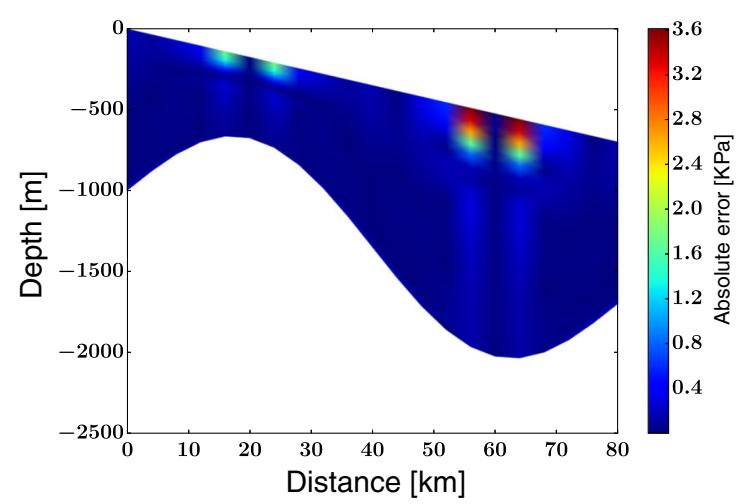

(c) Error of pressure, $p$

Fig. 6 Absolute error of Elmer/Ice in comparison to the numerical reference solution on the original case, on a $20 \times 20$ grid 
The expected local error (between two consecutive time steps) arising from errors in the velocity for an Euler forward time-stepping scheme is

$$
\begin{aligned}
s^{t+\Delta t} & =s^{t}+\Delta t\left(-\partial_{x} s\left(u_{x}+\epsilon_{x}\right)+u_{z}+\epsilon_{z}+a_{s}\right) \\
& =s^{t}+\Delta t\left(-\partial_{x} s u_{x}+u_{z}+a_{s}\right)+\Delta t\left(-\partial_{x} s \epsilon_{x}+\epsilon_{z}\right),
\end{aligned}
$$

where $\epsilon_{x}$ is the numerical error in the horizontal surface velocity $\left.u_{x}\right|_{z=s}$ and $\epsilon_{z}$ is the numerical error in the horizontal surface velocity $\left.u_{z}\right|_{z=s}$. The initial surface slope in the ISMIP-HOM B (and A) experiment is $0.5^{\circ}$, so that $\frac{\partial s}{\partial x}=-0.0087$. The error term in Eq. 14 is thus $\Delta t\left(0.0087 \epsilon_{x}+\epsilon_{z}\right)$. Considering that $\epsilon_{x} \leq 0.8$ and $\epsilon_{z} \leq 0.07$ in Fig. 6 and using a time step of $\Delta t=0.1$ year, the magnitude of the error is expected to be $0.1 \cdot 0.0087$. $0.8+0.1 \cdot 0.07 \approx 0.007$. The error in the horizontal surface velocity is significantly lower than 0.08 in all places except for at the singularity above the through, so overall, we expect that the errors in the vertical surface velocity will influence the accuracy of the free surface position more than the errors in the horizontal surface velocity.

\subsection{Experiment set-up}

To verify the above discussion and identify the errors propagating from the errors in the velocity field as separate from the errors arising from the discretization of Eq. 4 , we run two transient simulations, called Simulation I and Simulation II, spanning 10 years. Both simulations employs a forward Euler time-stepping scheme, with a fairly small time step of $\Delta t=0.1$ year to ensure stability. In Simulation I, the fine grid of $320 \times 80$ is used both for computing the velocity and pressure, and for solving the free surface evolution equation Eq. 4. In Simulation II, the fine grid is used only for solving the free surface evolution equation, and the $20 \times 20$ grid is used for computing the velocity field. As mentioned previously, the $20 \times 20$ resolution is equal to, or better than, a resolution (4 $\mathrm{km}$ cell size

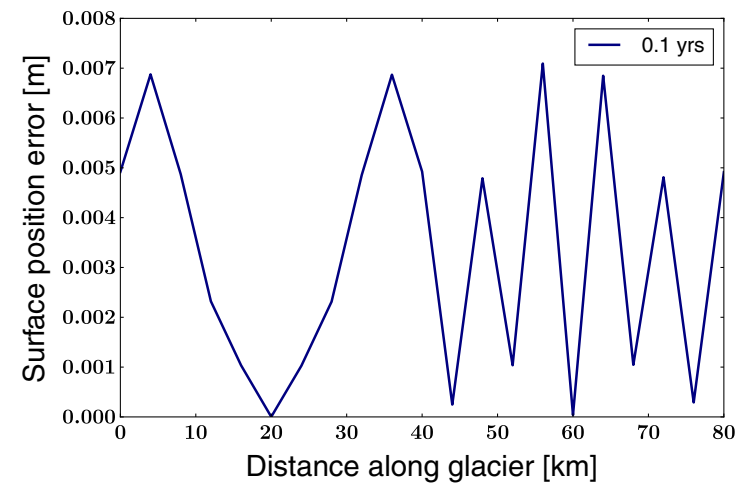

(a) Errors propagating from velocity inaccuracies after one time step in the horizontal with 20 vertical layers) of an ice-sheet simulation. By comparing the result of the two simulations, we can investigate the effect that the numerical errors in the velocity have on the free surface position.

We also repeated the experiments with a backward Euler time-stepping scheme with almost identical results. All the experiments are performed with Elmer/Ice as there is already an existing implementation of the free surface evolution.

\subsection{Results}

The difference between the free surface position in Simulation I and Simulation II is shown Fig. 7.

After one time step, the magnitude of the errors is indeed 0.007 , and the pattern of the errors in the free surface after one time step (Fig. 7a) corresponds to the pattern of the errors in the vertical velocity in Fig. 6 .

The local errors accumulate throughout the simulation, especially over the bump, see Fig. 7b. After 1 year, the error amounts to about $0.1 \mathrm{~m}$, which is on the order of magnitude of a typical net surface accumulation/ablation [13]. After 10 years, it is about $0.3 \mathrm{~m}$. The importance of the vertical velocity errors compared to the horizontal velocity errors is large for small surface slopes. For the ISMIP-HOM A and B experiment, the surface gradient is 0.0087 . In the Greenland SeaRISE data set $[10,13]$, it is never larger than 0.08 , and often much smaller.

Clearly, it is important to control the errors in the vertical velocity, in order to accurately predict ice surface position and ultimately the ice sheet volume. Despite this, the vertical velocity error is however rarely discussed, as the absolute vertical velocity error is lower than the absolute error in the horizontal velocity, and since the horizontal velocity is often of more interest to glaciologists than the vertical velocity. As we will see in the following section, an unfortunate choice of the GLS stabilization parameter has a larger impact on the vertical velocity than on the horizontal

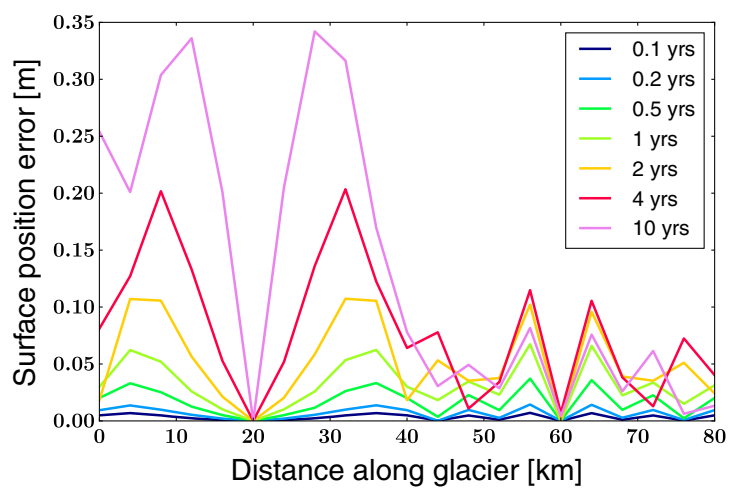

(b) Accumulation of propagated errors over 10 years

Fig. 7 Errors in the ice surface position due to velocity errors 
components. Especially considering the accuracy of the ice surface, it is important to pay attention to the choice of stabilization parameters.

\section{Sensitivity to the GLS stabilization parameter}

If the stability parameter $\tau_{\mathrm{GLS}}$ in Eq. 10 is not chosen carefully, significant errors will be introduced to the solution. For some cases, GLS stabilization also introduces artificial boundary conditions [19]. In this section, we perform experiments to assess whether the value in [31] (i.e., $\tau_{0}=1$ in Eq. 12), which was given for a Newtonian fluid on a simple domains and isotropic meshes, is a good choice for ice sheet simulations. To investigate the issues of over- and under-stabilization and artificial boundary conditions, and how sensitive these effects are to the stability parameter, we here perform experiments on three different experiment set-ups where we vary $\tau_{0}$. The three set-ups are as follows:

- $\quad$ The ISMIP-HOM domain

- An outlet glacier simulation

- Channel flow

In all simulations in this section, $h_{K}$ was defined as the minimum edge of the cell and the non-linear viscosity $\eta$ is used in the GLS stabilization parameter. A justification of these choices follows in Section 6.

\subsection{The ISMIP-HOM domain}

\subsubsection{Experiment set-up}

The domain and boundary conditions are set-up as in the previous ISMIP-HOM experiments. We run repeated simulations on the $20 \times 20$ mesh, varying between $\tau_{0}=10^{-4}$ and $\tau_{0}=10^{4}$, and measuring the accuracy of the solutions.
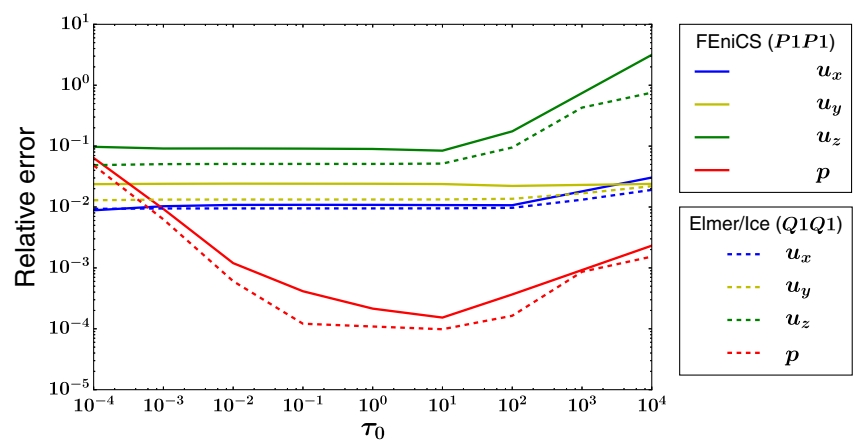

(a) Manufactured solutions as a reference

Fig. 8 The $L^{2}$-norm of the relative errors (compared to a manufactured solution (3D) and numerical reference solution (2D)) in velocity components and pressure for a GLS stabilization. The errors are
Despite our findings in Section 3 putting the manufactured problem in question, we here measure the errors both using a numerical reference solution, and on the manufactured problem. This is because the numerical reference solution will contain a stabilization term, and we use the manufactured solutions to ensure that this term does not influence our results. For the reference solution, we set $\tau_{0}=1$. We perform the experiments with both FEniCS and Elmer/Ice to ensure that the behavior of the stabilized problem is independent of implementation details.

\subsubsection{Results}

Figure 8 shows how the $L^{2}$-norm of the errors in the velocity components and pressure vary with $\tau_{0}$. For an under-stabilized problem, the error in the pressure is high due to spurious oscillations, while for a too large $\tau_{0}$, the stability terms alters the original problem significantly. The default parameter $\tau_{0}=1$ is a good choice while $\tau_{0}=$ 10 renders a slightly lower pressure error in FEniCS. The overall behavior is however very similar in FEniCS and Elmer/Ice, and therefore, all experiments from now on will be performed only in FEniCS for simplicity.

As seen in Fig. 8, an over-stabilized problem introduces relative errors mainly in the vertical velocity and pressure. Since the free surface position (and ultimately ice volume prediction) is sensitive to errors in the vertical velocity, the sensitivity of the vertical velocity to the stabilization parameter is unfortunate.

By considering the shallow ice approximation (SIA), we may gain some further understanding of why overstabilization mainly influence the vertical velocity component. Even though the SIA is a significant simplification of the problem, it is quite accurate for the ISMIP-HOM domain with $L=80 \mathrm{~km}[1,50]$. The SIA is based on the shallowness of the domain, so that one can assume $\frac{\partial(\cdot)}{\partial z}>>\frac{\partial(\cdot)}{\partial x}, \frac{\partial(\cdot)}{\partial y}$ and $u_{z}<<u_{x}, u_{y}$. According to the SIA, the $\mathfrak{p}$-Stokes

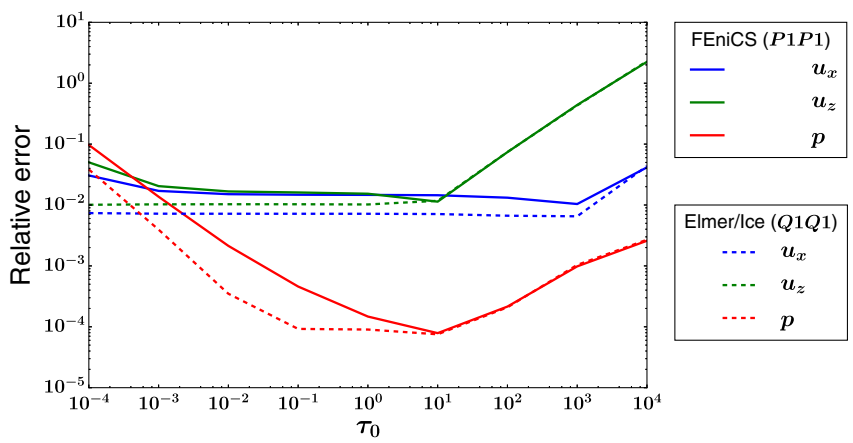

(b) Numerical reference solution

computed with FEniCS (solid lines, $P 1 P 1$ element) and Elmer/Ice (dashed lines, $Q 1 Q 1$ element) on a $20 \times 20 \times 20$ (for $2 \mathrm{D} 20 \times 20$ ) grid for varying $\tau_{0}$ (panel (a) and (b)) 
system of Eq. 3 (in two dimensions) can, together with the incompressibility constraint, be reduced to

$$
\begin{aligned}
-\frac{\partial p}{\partial x}+\frac{\partial}{\partial z}\left(\eta \frac{\partial u_{x}}{\partial z}\right) & =0 \\
-\frac{\partial p}{\partial z} & =\rho g \\
\frac{\partial u_{x}}{\partial x}+\frac{\partial u_{z}}{\partial z} & =0
\end{aligned}
$$

In the SIA equations, since the pressure is given by the vertical momentum balance of Eq. 15b, the horizontal velocity $u_{x}$ is thus solely determined by the horizontal momentum balance of Eq. 15a. Using the pressure and horizontal velocity, the vertical velocity can then be solved for in Eq. 15c. The stabilization term in Eq. 10 is added to the balance of mass Eq. 15c, and since the horizontal velocity is already restricted by the horizontal momentum balance, the vertical velocity will balance any alterations introduced by the stabilization parameter. The sensitivity of the vertical velocity is thus largely related to its modest magnitude. For thicker domains, where the SIA does not hold, the sensitivity to $\tau_{0}$ is more equally distributed among the velocity components. Note that the non-linearity of the viscosity is not important here, and also for a linear viscosity the errors associated with over-stabilization are larger in the vertical component.

\subsection{An outlet glacier}

In this section, we study a more realistic set-up than the ISMIPHOM problem. In glaciology, a common problem is to model a single outlet glacier which is part of a larger ice sheet. Inlet velocity boundary conditions are typically given by a coarse mesh simulation of the whole ice sheet using the Stokes equation, or a cheaper method like the shallow ice approximation [35] or the Blatter-Pattyn [15, 49] formulation of the system. This set-up introduces two separate complications that we study: (1) a more complicated geometry with a glacier front and (2) a more involved lateral (and in one experiment basal) boundary conditions.

\subsubsection{Experiment set-up}

We model the front of a two-dimensional glacier specified by a Vialov type surface profile [59] underlain by a bumpy bed. The surface $(s)$ and bed $(b)$ of the entire ice sheet are defined by

$$
\begin{aligned}
s(x) & =H\left(1-\left(\frac{|x|}{L}\right)^{\frac{n+1}{n}}\right)^{\frac{n}{2 n+2}}+200, \\
b(x) & =150 \cos \left(\frac{10 \pi|x|}{L}\right)\left(1-\cos \left(\frac{\pi x}{2 L}\right)\right) \\
& +250 \cos \left(\frac{\pi x}{2 L}\right)+25 \cos \left(\frac{80 \pi|x|}{L}\right),
\end{aligned}
$$

where the dome is at $x=0$, the radius of the ice sheet is $L=300 \mathrm{~km}$, the maximum height is $H=2000 \mathrm{~m}$ and as before, $n=\frac{1}{\mathfrak{p}-1}=3$. We focus on the region stretching from $x=275 \mathrm{~km}$ to the glacier front at $x=300 \mathrm{~km}$.

To produce an inflow boundary condition at $x=275 \mathrm{~km}$, we first simulate the entire ice-sheet simulation on a coarse mesh. This coarse mesh is an extruded $160 \times 20$ mesh which is equidistant in the horizontal direction. The bedrock undulations with the highest frequency (i.e., the last term in Eq. 16) cannot be resolved properly on the coarse mesh and are therefore excluded when generating the inflow conditions.

The mesh used for the front simulation is a horizontally refined mesh with horizontal resolution of $100-850 \mathrm{~m}$ (see Fig. 15b). This is done to resemble a practical simulations, as it is common to apply horizontal plane mesh refinement near glacier fronts. Horizontal mesh refinement will be discussed further in Section 6.1. At the glacier front, we have specified a natural, stress-free condition.

Three different scenarios are simulated. In the first scenario, no-slip conditions are applied at the base, as in the ISMIP-HOM experiment. In the second scenario, more realistic conditions are considered by applying a sliding law at the base. The coefficient of basal traction $\left(\beta^{2}\right)$ is

$\beta^{2}=a e^{\frac{-x^{2}}{2 b^{2}}}+c$

The parameters of the sliding field, $a=2.7 e^{4}, b=5.8 e^{4}$, $c=650$, are the result of a least-squares fit to the sliding field of a transect on the south western side of the Greenland ice sheet (symmetrized to fit the Vialov scenario). In the third scenario, we explore how the non-linear material influence the accuracy of the GLS stabilization. In that setup, we use three different constant viscosities, $\eta_{\text {high }}=1 e^{8}$, $\eta_{\text {mid }}=1 e^{6}, \eta_{\text {low }}=3 e^{4}(\mathrm{~Pa}$ a), which span the viscosity range simulated in the non-linear setting. The high value is reached in two areas near the ice surface, approximately around $x=282 \mathrm{~km}$ and $x=289 \mathrm{~km}$, i.e above two of the throughs. The lowest value is reached at the glacier front.

Bearing the findings of Section 4 in mind, we focus our attention on the surface velocity in the vertical direction and how this changes with the type of stabilization method and size of stabilization parameter. Note that although the surface gradient is steep at the very edge of the glacier it is still only 0.05 at $x=297 \mathrm{~km}$ and 0.09 at $x=$ $299 \mathrm{~km}$, so that the discussion in Section 4 is still relavant. To study the impact of the stabilization parameter, we vary $\tau_{0}$ and measure changes in the vertical velocities when compared to an unstabilized velocity field. While this approach does not allow us to measure exact errors, it does show the sensitivity to the stabilization parameter. The unstabilized velocity field is non-oscillatory. In the figures, we only present simulations where $\tau_{0}$ was large enough to 
stabilize the pressure. To determine when the pressure was non-oscillatory, we first measure the relative change in the pressure variable as the stabilization parameter increases. We do this by taking the norm of the difference of two pressure solutions corresponding to two subsequent values of $\tau_{0}$, divided by the norm of the first solution (i.e., a shifting relative error, $\frac{\left|p_{\tau_{i+1}}-p_{\tau_{i}}\right|}{\left|p_{\tau_{i}}\right|}$ ). When this value change less than $10^{-2}$, it is deemed that increasing the stabilization parameter does not significantly change the pressure variable, i.e., that the pressure is stabilized. In addition, we also monitor the relative error compared to the pressure solution of the same simulation performed with the stable $P 2 P 1$ element. When this value drops below $2.5 e^{-3}$, the pressure is essentially oscillation-free. Both of the above methods corresponded well to the "eye-ball norm," which served as a (slightly conservative) control.

\subsubsection{Results}

The result for the first scenario with frozen basal conditions and a non-linear viscosity is presented in Fig. 9. The smallest $\tau_{0}=0.1$ shown is the minimal value that resulted in an acceptable, oscillation-free, pressure field. Firstly, we note that setting an inflow boundary condition makes the pressure more prone to oscillations, specifically near the inlet. Secondly, any value above $\tau_{0}=0.1$ introduces oscillations in the velocity at the front of the glacier. Additional simulations of the entire ice sheet shows that this is not related to the inlet boundary condition, but occurs also for simulations of the entire ice sheet. Because of such an over-stabilization of the vertical velocity, the default GLS parameter as given in [31] (obtained for $\tau_{0}=1$ ) does not

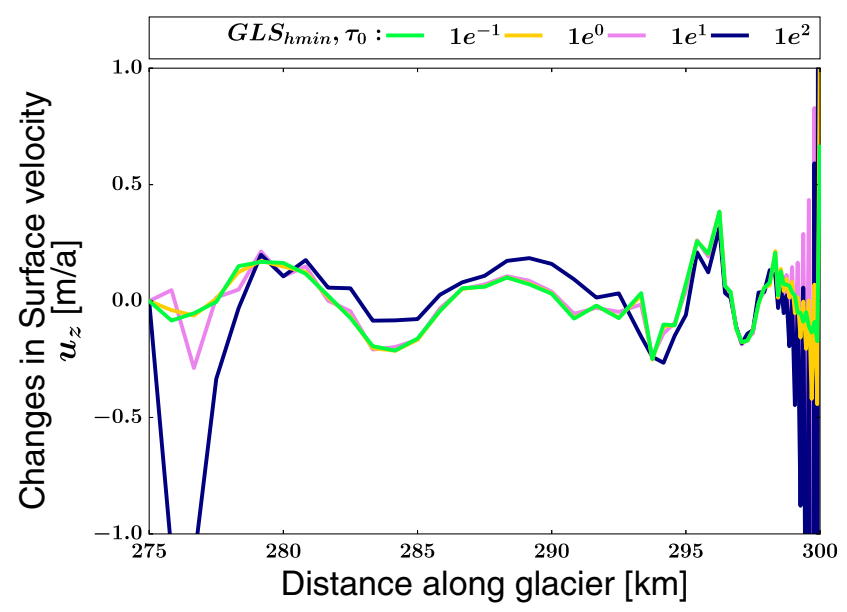

Fig.9 Vialov frozen scenario. Changes in vertical velocity for GLS for different values of $\tau_{0}$, compared to an unstabilized velocity solution. The values of $\tau_{0}$ have been chosen such that any smaller values produced oscillations in the pressure field

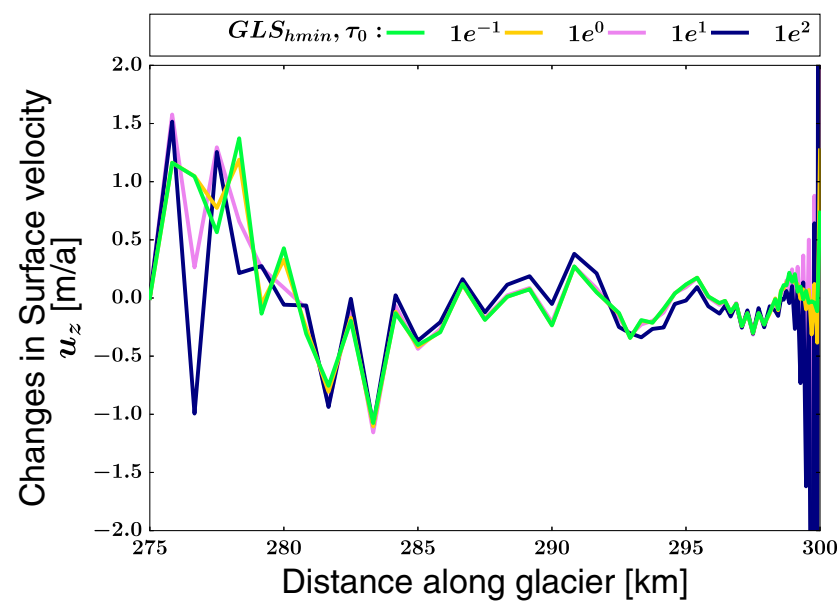

Fig. 10 Vialov sliding scenario. Changes in vertical velocity for GLS for different values of $\tau_{0}$, compared to an unstabilized velocity solution. The values of $\tau_{0}$ have been chosen such that any smaller values produced oscillations in the pressure field

seem to be an appropriate choice. The two effects (inlet oscillations and frontal oscillations) together narrow the range of possible stability parameters. Note that there are also some variations in the interior, in the two areas with high viscosity, around $x=282 \mathrm{~km}$ and $x=289 \mathrm{~km}$. The results for the second scenario with basal sliding and a nonlinear viscosity is shown in Fig. 10) (note the change of axis scale). Introducing sliding does not affect the frontal oscillations, but increases the instabilities at the inlet, further increasing the risk of over-stabilization in realistic icesheet simulations. Another issue is that, compared to other techniques, and also the $P 2 P 1$ element, it appears that the stable pressure is higher for the GLS method even though

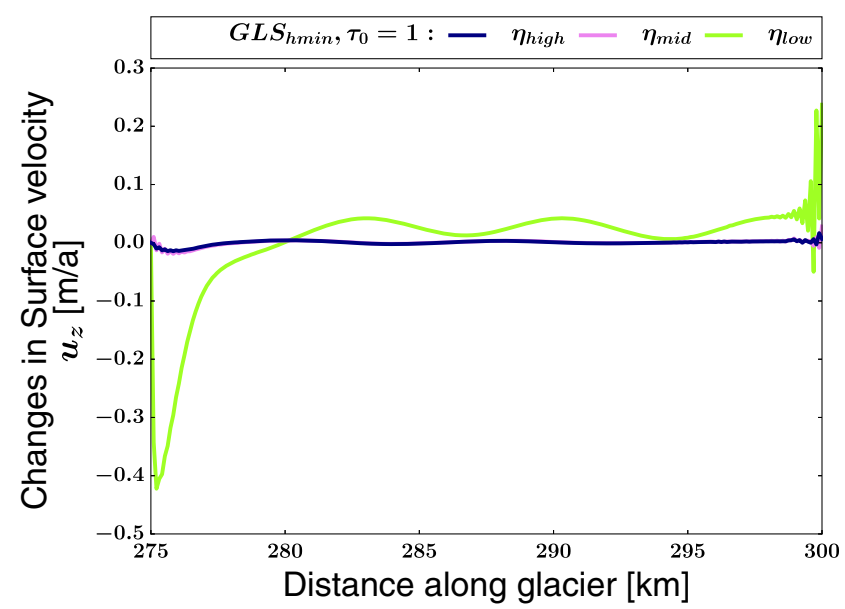

Fig. 11 Vialov frozen scenario with linear viscosity. Changes in vertical velocity for GLS for different viscosity values: $\eta_{\text {high }}=1 e^{8}$, $\eta_{\text {mid }}=1 e^{6}, \eta_{\text {low }}=3 e^{4}$ ( $\mathrm{Pa}$ a), compared to an unstabilized velocity solution. In all simulations $\tau_{0}=1$ 
it is oscillation-free. In Fig. 11, the results of the third scenario with frozen basal conditions and a linear flow law is shown. Here, $\tau_{0}=1$ and the viscosity values are varied. The results indicates that the over-stabilization becomes more prominent for lower viscosity values, emphasizing the importance of a robust stabilization method in areas of high deformation, e.g., close to the front. Noteworthy is also that the variations in the interior largely vanish for constant viscosities. This indicates that viscosity gradients are influencing the accuracy of the GLS method. Possibly, the variation of the oscillations can be connected to the consistency of the method. Due to the linear elements, the viscous terms are dropped in the GLS term, so that the stability term tends to enforce $\nabla p=\rho g$. In the interior, the SIA is fairly accurate, so that $\nabla p \approx \rho g$. At the front and in areas with high viscosity gradients, however, $\nabla p=\rho g$ is not true and the stability term has a higher influence. Indeed, when studying conservation of mass, it is least accurate at the front. A related reasoning lies behind the concept of artificial boundary conditions, which may be more related to the inlet oscillations. Artificial boundary conditions is the topic of the next section.

Remark: For all experiments, the changes in the horizontal surface velocity were of the same absolute magnitude at the front. Relating to the the discussion in Section 5, the SIA argument does not hold at the glacier front but does hold in the main part of the glacier.

\subsection{Poiseuille flow - artificial boundary conditions}

In this section, we focus on artifacts at the boundary due to artificial boundary conditions. It is a known problem of GLS stabilization that it introduces the artificial boundary condition

$$
\int_{\partial \Omega} \tau_{\mathrm{GLS}}\left(\partial_{n} p_{h}-\rho g \cdot n\right) d s \approx 0,
$$

where $\partial_{n}$ denotes the normal derivative. [11, 29] showed that such artificial boundary conditions can have significant effect on the numerical solution of the pressure, using simulations of Newtonian so-called Poiseuille flow as an example.

\subsubsection{Experiment set-up}

To show the influence of artificial boundary conditions, we perform two-dimensional Poiseuille flow experiments with ice, i.e., with a non-linear viscosity instead of the linear of $[11,29]$. Poiseuille flow is a pressure driven flow in a channel. The simulations are performed in the horizontal plane so that the body force is zero $(\rho g \cdot n=0)$. The analytical solution is given by

$$
\begin{aligned}
\left(u_{x}, u_{y}\right) & =\left(\frac{2^{\frac{1-n}{2}}}{n+1}\left(\frac{p_{x}}{\eta_{0}}\right)^{n}\left(R^{n+1}-|R-y|^{n+1}\right), 0.0\right) \\
p & =p_{x}(L-x),
\end{aligned}
$$

where $n=3$ and $\eta_{0}$ are the parameters described in Eq. 2, $L=0.5 \mathrm{~km}$ is the horizontal extension of the domain, $R=$ $0.5 \mathrm{~km}$ is the radius of the channel, and $p_{x}=200 \mathrm{~Pa} \mathrm{~m}^{-1}$ is the $x$-directional gradient of the pressure. We prescribed the analytical solution as an inflow velocity at $x=0$ and no-slip boundary conditions at $y=0$ and $y=2 R$. As a outflow condition we prescribed a Neumann condition with the additional requirement that the vertical velocity be zero. Since the pressure is not unique, we set a point Dirichlet condition at $(x, y)=(L, 0)$ where the pressure equals zero. The simulations were preformed on a $20 \times 20$ grid, with $h_{K}$ set as diameter of the cell (maximum cell edge length for triangles). However, note that in this horizontal plane simulation the mesh is isotropic so that $h_{\min } \sim h_{\max }$.

\subsubsection{Results}

The results are shown in Fig. 12. We included the analytical solution as a reference.

The pressure contours in (Fig. 12a) bends towards the edges, which is the typical effect of artificial boundary conditions. Hence, the choice of $\tau_{0}=1$ is large enough to stabilize the pressure, but too large to avoid a significant effect of the artificial boundary condition.

Another interesting feature of Fig. 12a is the spike of the pressure contours observed along $y=500 \mathrm{~m}$ in Fig. 12a due to a singularity in the viscosity. The problem is not easily amendable by varying the critical shear rate that regularizes the viscosity. The spikes decrease as $\tau_{0}$ increases, but it is not possible to increase the stability parameter enough to visibly eliminate the pressure spike without over-stabilizing the solution grossly, see Fig. 12b. The problem becomes less severe with mesh refinement, but for practical meshes the problem remains. The simulation was also performed with the inf-sup stable $P 2 P 1$ element, which indicated that the pressure was affected by the singularity in a similar way to the GLS method, although to a lesser extent.

Poiseuille flow does remind of a horizontal plane simulation of a glacier in a confined bay with low basal friction. Tentative experiments indicate that the pressure field can be affected in such glacier simulations. However, further studies are need to determine whether this is due to setting inflow boundary conditions of due to overstabilization. Note that in a three-dimensional simulation $\rho g \cdot n \neq 0$. In fact, for a three-dimensional simulation, 


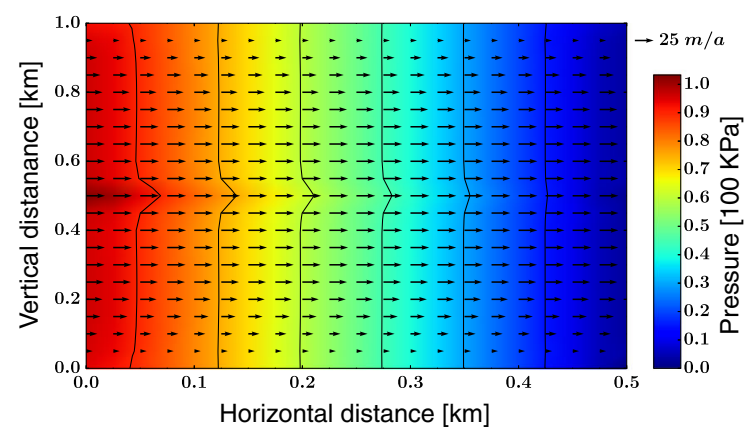

(a) GLS method, $\tau_{0}=1$

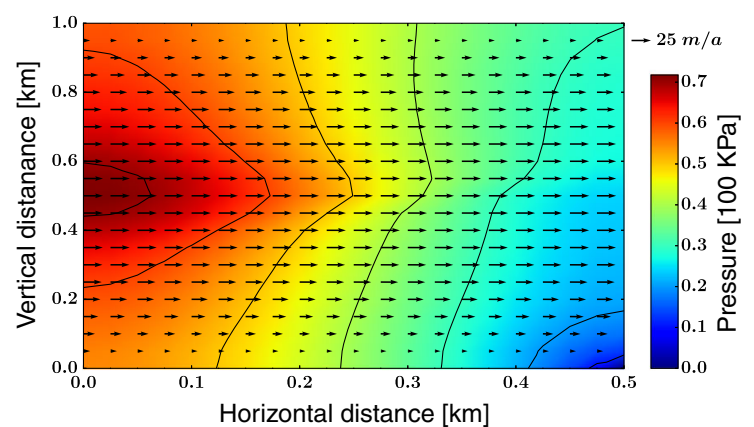

(b) GLS method, $\tau_{0}=100$

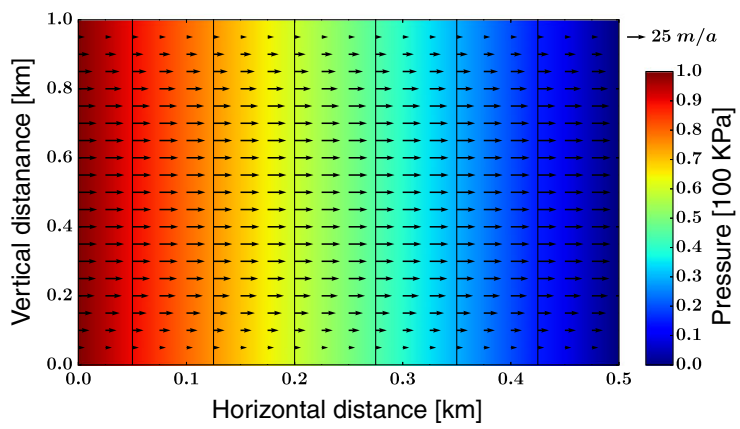

(c) Analytical solution

Fig. 12 Poiseuille flow stabilized with the GLS method and the analytical solution. Stabilization parameter is $\tau_{0}=1$ (left column) or $\tau_{0}=100$ (right column), $h_{K}$ is taken to be maximum cell edge or facet. Velocity is depicted as black arrows and pressure as color with black lines as pressure contours

the artificial boundary condition will enforce a hydrostatic pressure near boundaries.

\section{Stability parameter choices}

In previous sections, we explored the effects of over- and under-stabilization by varying the parameter $\tau_{0}$. In practice, $\tau_{0}$ is chosen as 1 and such over- or under-stabilization would instead be due to that (1) the method or the analysis of [31] is not valid for the specific problem, as in the case of the outlet glacier, or (2) that the variables in $\tau_{\mathrm{GLS}}$ (i.e., $h_{K}$ and $\eta$ ) of Eq. 12 are not set appropriately. In this section, we explore the second case. So far, we defined $h_{K}$ as the minimum edge length (apart from in the Poiseuille case) and let the viscosity vary with the velocity. This is not always the case in all ice-sheet models. In this section, we try alternative approaches to gain insight in how $h_{K}$ and $\eta$ should be used in $\tau_{\mathrm{GLS}}$.

\subsection{The cell size $h_{K}$}

While standard stabilization techniques were originally developed for isotropic meshes, a typical mesh in an ice-sheet simulation can render elements with an aspect ratio as high as 200 . The aspect ratio affects the accuracy and stability of the numerical solution [55]. Given the flat elements, and that analysis and stabilization parameter choices for anisotropic meshes is still a relatively unexplored corner of numerical analysis [42], it is important to consider the definition of the cell size $h_{K}$ in $\tau_{\text {GLS }}$ in Eq. 10.

If the cell size is defined as the minimum edge length, $h_{\min }$, (see, e.g., [57]) the stabilization parameter will scale with the topography of the domain. However, since most meshes used in glaciology are of extruded type, refinements are commonly applied at the footprint level (horizontal refinement) $[33,44$, 54] according to some measure such as velocity changes. Such a refinement will have little effect on the size of $h_{\text {min }}$ while the aspect ratio of the cells may vary substantially.

Alternatively, one can set $h_{K}$ as the diameter of the cell (denoted as $h_{\max }$ ), i.e., as the length of the maximum edge for a triangle/tetrahedron or the largest diagonal of the cell for a rectangle/hexahedron. This measure is the default choice on isotropic meshes and allows for a potential horizontal refinement to affect the stabilization parameter. However, this definition may cause problems in glaciology, since for an extruded mesh with anisotropic elements such as in Fig. 2, the diameter does not account well for the variations in topography. Since the dominating effect of the stabilization is in the vertical, it could lead to overstabilization for elements with high aspect ratio on a highly varying topography. 
Another possibility is to use methods developed specifically for anisotropic meshes. In [14], a GLS stabilization for high aspect ratios is developed. The technique is based on using he Jacobian matrix, $J_{K}$, of the affine transformation from the reference element $\hat{K}$ to the current element $K$. The modified stabilization for our case then becomes

$$
\begin{aligned}
& S_{J}\left(\left(u_{h}, p_{h}\right),\left(v_{h}, q_{h}\right)\right) \\
& =-\sum_{K \in T_{h}} \frac{m_{K}}{8 \eta}\left(J_{K} \cdot\left(-\nabla \cdot S(u)+\nabla p_{h}\right),\right. \\
& \left.J_{K} \cdot\left(-\nabla \cdot S(v)+\nabla q_{h}\right)\right)_{K}, \\
& F_{J}\left(\left(v_{h}, q_{h}\right)\right)= \\
& -\sum_{K \in T_{h}} \frac{m_{K}}{8 \eta}\left(J_{K} \cdot \rho g, J_{K} \cdot(\nabla \cdot S(v))+\nabla q_{h}\right)_{K} .
\end{aligned}
$$

Another method for anisotropic meshes was developed in [48], which is based on a singular value decomposition of the Jacobian. The method is equivalent to using the minimum cells size, if the meshes are not sheared. The shearing of the elements in ice-sheet modeling is not large enough to justify the use of this method.

We now make numerical experiments on the ISMIPHOM problem and the outlet glacier of Section 5.2 to compare the effect of using (1) a standard GLS formulation $h_{K}=h_{\max }$, (2) a standard GLS formulation $h_{K}=h_{\min }$, and (3) the anisotropic stabilization of Eq. 19.

\subsubsection{The ISMIP-HOM domain - horizontal mesh refinement}

Experiment set-up We simulate the ISMIP-HOM B domain on five different meshes with different horizontal resolution, $20 \times 20,40 \times 20,80 \times 20,160 \times 20$, and $320 \times 20$, and the three different approaches to the anisotropic meshes. For each mesh and method, we also vary $\tau_{0}$ to show how the appropriateness of the default parameter $\tau_{0}=1$ varies.

For the experiments in this section, we used FEniCS and the numerical reference solution on the ISMIP-HOM B problem.

Results The results are shown in Fig. 13. As we saw in previous sections, the default value of $\tau_{\mathrm{GLS}}\left(\tau_{0}=1\right)$ is appropriate on the ISMIP-HOM domain, and even optimal on lower aspect ratios. The value of $h_{\max }$ is much larger than $h_{\min }$, and the stability parameter needs to be decreased if $h_{K}=h_{\max }$, in order to balance the larger element measure.

For $h_{K}=h_{\max }$, a horizontal refinement of the mesh (decrease in $h_{\max }$ ) leads to an increase in the optimal values of $\tau_{0}$, since the quadratic decrease in the size of $\tau_{\mathrm{GLS}}$ with refinement is too rapid (Fig. 13a and b). Defining the cell size as $h_{\text {min }}$ leads to an decrease in the optimal $\tau_{0}$ (Fig. $13 \mathrm{c}$ and d), since the size of $h_{\min }$ and thereby $\tau_{\mathrm{GLS}}$ is not affected by horizontal refinement.
The variability of the optimal value of the stability parameter with mesh size makes it harder to find such a value on a mesh with varying cell size. For $h_{K}=h_{\max }$, this could affect the pressure variable by not achieving the expected increase of accuracy as the mesh is refined, while for $h_{K}=h_{\mathrm{min}}$, it could result in slightly over-stabilizing the velocity and pressure in finer cells. For simple problems like the ISMIP-HOM domain this effect is not large, however, it may be more severe for topographies more complex than the ISMIP-HOM experiment on a $80 \mathrm{~km}$ long domain. The cell size definition on highly varying topographies and meshes is an interesting topic to explore further in future studies.

The isotropic stabilization term clearly poses some problems on anisotropic meshes. However, as can be seen in Fig. 13e, f, the anisotropic GLS stabilization does not seem to be of any noteworthy improvement compared to the regular GLS method, and due to this, we choose to not discuss it further. We performed the same simulation using a linear flow law with similar results.

\subsubsection{The outlet glacier experiment}

Experiment Set-Up To investigate the impact of cell size definition for a more realistic scenario, we here repeat the outlet-glacier simulation of Section 5.2 with $h_{K}=h_{\max }$ instead of $h_{K}=h_{\min }$.

Results The result is presented in Fig. 14. As expected, the feasible values of $\tau_{0}$ are lower than for $h_{K}=h_{\text {min }}$ (compare to to $h_{K}=h_{\min }$ in Fig. 9). For the best choice of the stabilization parameter $\left(\tau_{0}=10^{4}-10^{-3}\right)$, the accuracy at the front is comparable to the minimum edge length case, but increasing $\tau_{0}$ leads to over-stabilization more rapidly than for $h_{K}=h_{\min }$. Furthermore, it seems that the maximum edge length, even for moderate values of $\tau_{0}$, introduces larger changes than $h_{\min }$ in the vertical velocity inland, far from the front and inlet.

\subsection{The viscosity $\eta$}

The GLS stabilization in [31] was developed for Newtonian fluids, where $\eta$ is constant. In Elmer/Ice, the non-Newtonian nature of ice is accounted for by allowing the viscosity to vary also in the stabilization parameter. An alternative approach is to simply use a constant approximation of the ice viscosity, $\eta_{\text {lin }}$, in the stabilization parameter. This approach has been used by [22], where the authors reported that this choice resulted in better numerical stability. Hirn [38], however, found that the convergence of the iterative solvers was better when the stability parameter varied with the viscosity.

We denote the constant viscosity stabilization parameter by $\tau_{\text {lin }}$, using the common choice of $\eta_{\text {lin }}=10^{14} \mathrm{~Pa} \mathrm{~s}$. 


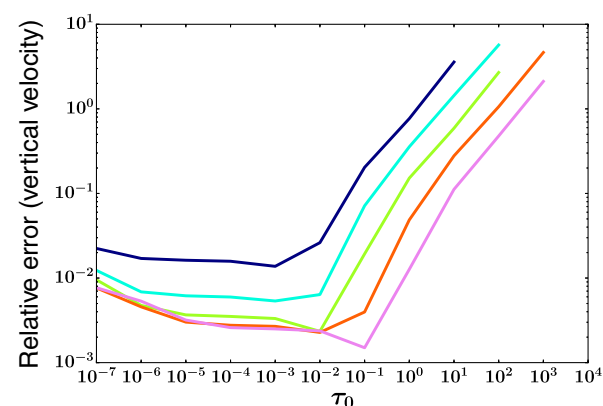

(a) Vertical velocity, $h_{k}=h_{\max }$

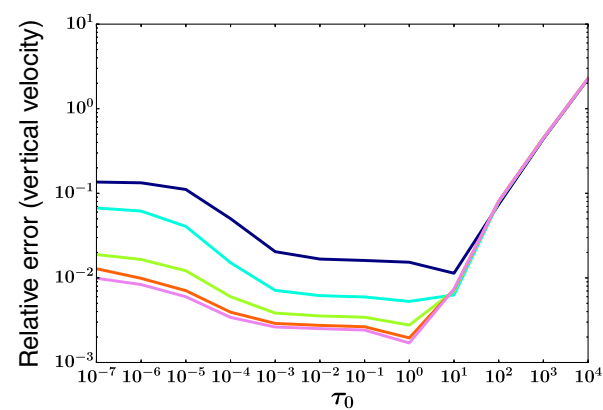

(c) Vertical velocity, $h_{k}=h_{\min }$

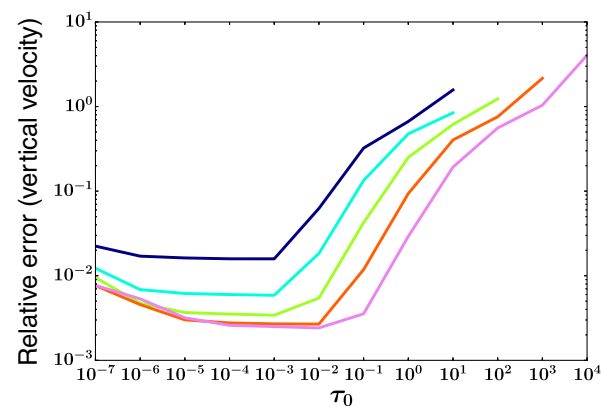

(e) Vertical velocity, anisotropic formulation
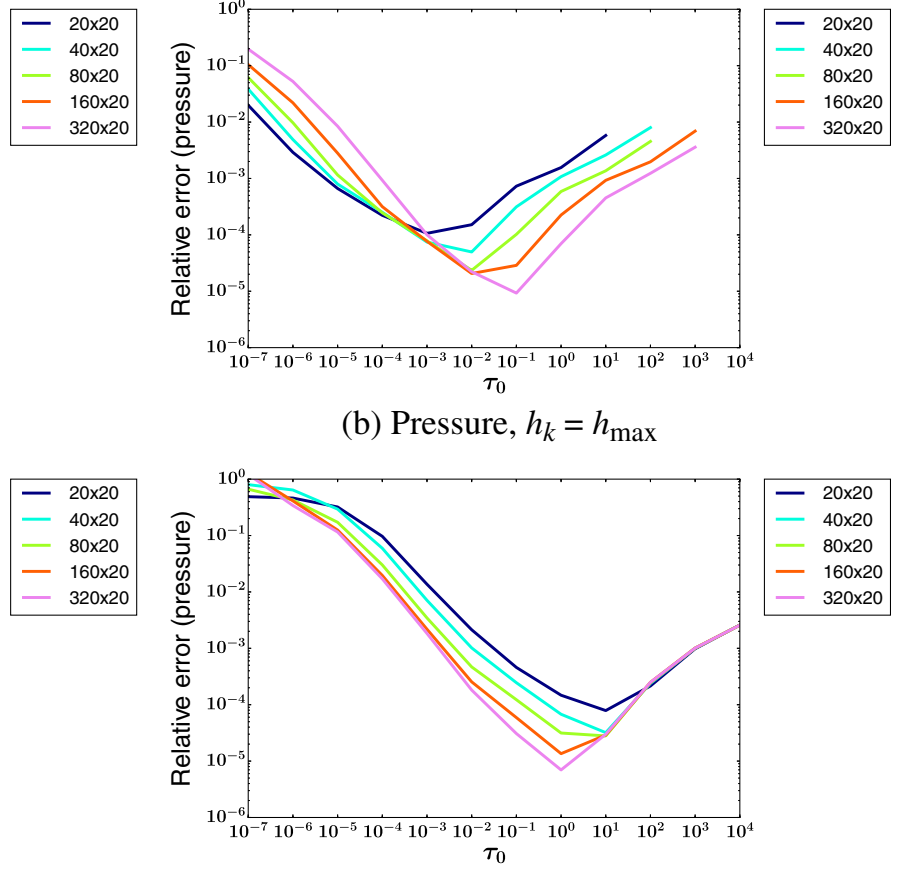

(d) Pressure, $h_{k}=h_{\text {min }}$
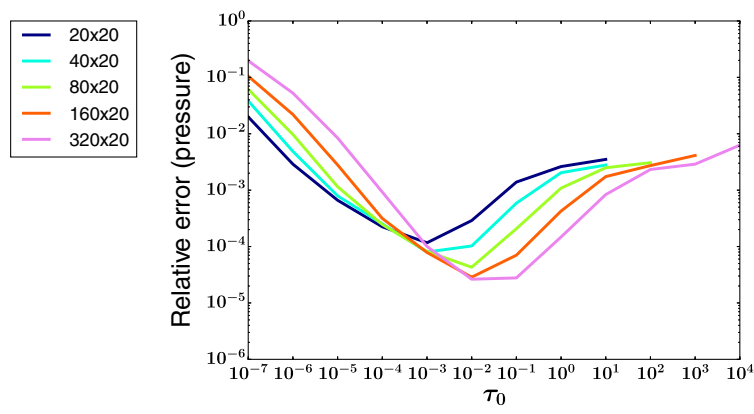

(f) Pressure, anistropic formulation

Fig. 13 Dependency of relative errors of vertical velocity and pressure on $\tau_{\mathrm{GLS}}$ and the horizontal refinement of the ISMIP-HOM A domain using isotropic stabilization with $h_{K}=h_{\max }, h_{K}=h_{\min }$, or using an anisotropic formulation

Figure 15 shows the ratio of $\tau_{\mathrm{GLS}} / \tau_{\text {lin }}$ for the ISMIP$\mathrm{HOM}$ and outlet glacier domain. The magnitude of $\tau_{\mathrm{GLS}}$ is an order of magnitude higher at the bed, but perhaps more importantly, $\tau_{\text {lin }}$ is two (ISMIP-HOM) or three (outlet glacier) orders of magnitude higher than $\tau_{\mathrm{GLS}}$ at points close to the surface where deformation is low and viscosity is high. This may lead to over-stabilization and thereby high errors in the vertical velocity. Since it occurs at the surface this will effect the accuracy of the free surface position. Further comparison studies between the two methods are needed but out of the scope of this work.

\section{Alternative stabilization techniques}

We have seen so far how over-stabilization may occur from GLS stabilization, either because the method and analysis of [31] is not appropriate for the set-up, or because stabilization parameters are not defined correctly. The model experiments that we have used are relatively simple, and these effects may be amplified on a full scale ice sheet simulation. Therefore, a more robust method would be favorable. In this section, we compare GLS to other types of stabilization methods. We will mainly focus on how the pressure and and in particular vertical surface velocities change with the size of the stabilization parameter. The implemented methods include a pressure penalty method (PP), an interior penalty (IP), pressure global projection (PGP), and a linear local pressure stabilization (LPS). The PP method is very simple and has already been used in glaciology. The latter three have to our knowledge not been used in glaciology yet, but are promising as they have been reported as less sensitive to over-stabilization 


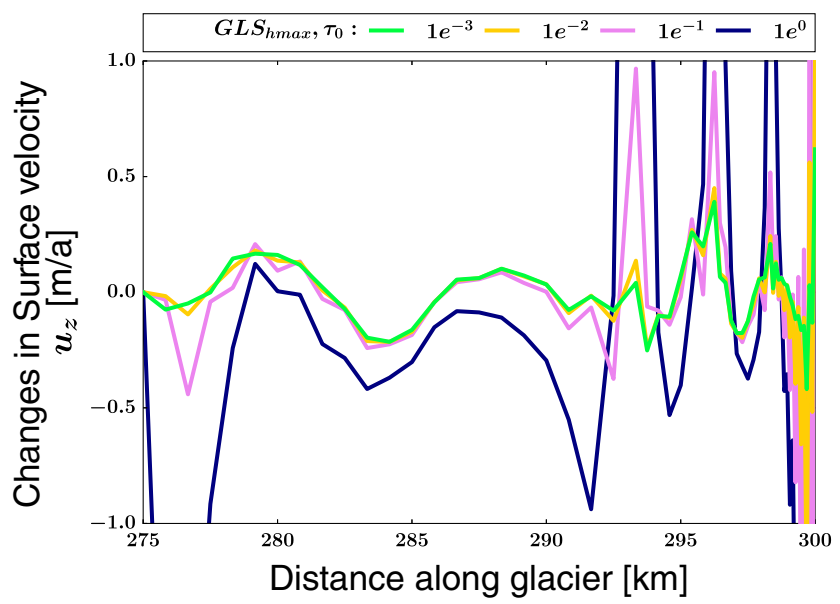

Fig. 14 Changes in vertical velocity for GLS for different values of $\tau_{0}$ with $h_{K}=h_{\max }$, compared to an unstabilized velocity solution. The smallest value of $\tau_{0}$ shown is the smallest $\tau_{0}$ that ensured a stable pressure field

and viscosity changes for some scenarios [18]. After a brief description of these alternative stabilization methods, we repeat the stabilization parameter sensitivity experiment from Section 5. Based on the performance of each method, we select the IP and PGP methods to redo the outlet glacier and finally examine how the IP method performs in the non-linear Poiseuille flow.

The stabilization methods in this section have all been implemented in FEniCS, which allows for easy implementation of new forms. The elements are triangles (for 2D) or tetrahedrons (for 3D). Since second-order derivatives are identically zero on such elements, we have omitted them in the descriptions below. In all of the below implemented stabilization methods, we use the constant viscosity stabilization parameter $\tau_{\text {lin }}=\tau_{0} \frac{m_{K} h_{K}^{2}}{8 \eta_{\text {lin }}}$, using either $h_{K}=h_{\min }$ or $h_{K}=h_{\max }$. The reason for this choice is that in the above methods, the stabilization parameter is defined to be some user-defined constant times $h_{K}^{2}$, but not explicitly dependent on the viscosity. To facilitate the comparison of the different methods, we then choose the user-defined part of the stabilization parameter to be the same and similar in size to the parameter used in the GLS method. Unless the method results in an augmented system (i.e., PGP), the stabilization terms are added to the left-hand side of Eq. 10.

\subsection{Description}

\subsubsection{Pressure penalty method}

This minimalistic stabilization method is penalizing pressure gradients by adding the stabilization term

$-\sum_{K \in T_{h}} \tau_{\operatorname{lin}}\left(\nabla p_{h}, \nabla q_{h}\right)_{K}$

The method is very easy to implement and has been used for glaciological applications in [60].

\subsubsection{Interior penalty method}

In [23] the authors penalized pressure gradients on the interior edges of the domain to stabilize the linear Stokes equations. The stabilization term for a viscous problems is

$-\sum_{K \in T_{h}} \tau_{\operatorname{lin}} \int_{\partial K}\left[n \cdot \nabla p_{h}\right]\left[n \cdot \nabla q_{h}\right] d s$,

where [.] indicates the jump over an interior edge $\partial K$.

In a study of Poiseuille flow for a Newtonian fluid, Burman and Hansbo [23] show that this method does not introduce artificial boundary conditions as strongly as the GLS method does.

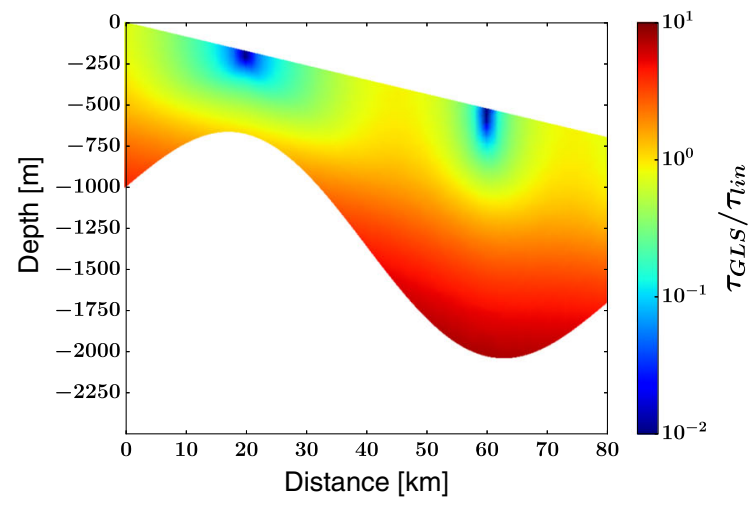

(a) ISMIP-HOM A domain

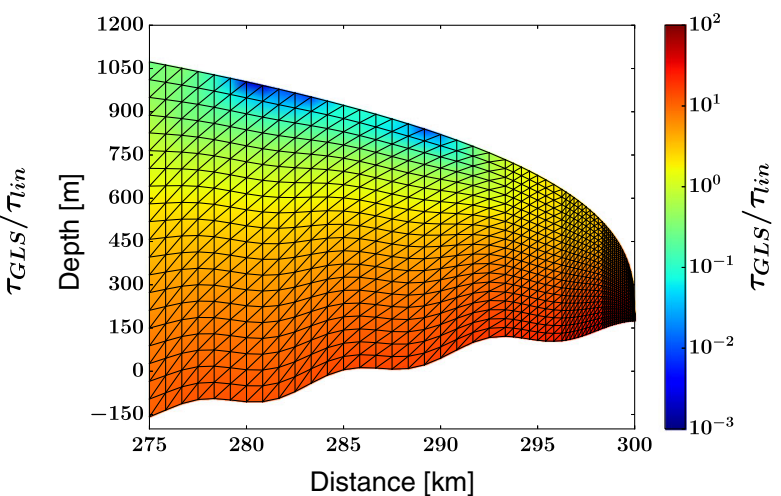

(b) Vialov type domain on refined mesh

Fig. 15 Ratios of the stabilization parameters $\tau_{\mathrm{GLS}} / \tau_{\text {lin }}$ for the domains used in this study 


\subsubsection{Pressure global projection method}

In [27], the gradient of the pressure is projected onto the velocity space, resulting in an augmented system:

$$
\begin{aligned}
& A\left(u_{h}, v_{h}\right)+B\left(v_{h}, p_{h}\right)=F\left(v_{h}\right) \quad \forall v_{h} \in V_{h}, \\
& -B\left(u_{h}, q_{h}\right)+\tau_{l i n}\left(\nabla p_{h}-\xi_{h}, \nabla q_{h}\right)=0 \quad \forall q_{h} \in Q_{h}, \\
& -\left(\nabla p_{h}, \eta_{h}\right)+\left(\xi_{h}, \eta_{h}\right)=0 \quad \forall \eta_{h} \in \stackrel{\circ}{h}_{h},
\end{aligned}
$$

where $\dot{V}_{h}$ is the same space as the velocity space but without boundary conditions. The method introduces an additional variable, $\xi$, and therefore increases the computational cost.

\subsubsection{Local projection stabilization method}

Related to the pressure projection method, is a method by [11] where the gradient of the pressure is projected onto a space of piece-wise constants defined on a coarser mesh. In the coarse mesh every element $M$ is a patch consisting of elements $K$ from the original mesh. The stabilization term is

$\tau_{\operatorname{lin}}\left(\nabla p_{h}-\overline{\nabla p_{h}}, \nabla q_{h}\right)=0 \quad \forall q_{h} \in Q_{h}$

where $\overline{\nabla p_{h}}$ is the onto the coarse mesh projected pressure gradient. Compared to the GLS, the LPS reduces the coupling between pressure and velocity but the stencil for the pressure variable increases due to the evaluation of the projected gradient on the coarser mesh.

In this study, each $M$ is subdivided into 16 triangles with similar properties as the parent patch. To avoid the larger stencil for the pressure, we use the pressure solution from the previous Newton iteration to solve for the projected pressure gradient.

One of the benefits of the LPS method is that there exists a rigorous adaptation to the $\mathfrak{p}$-Stokes equations [39], which is advantageous for three dimensional simulations or low regularity data. The method has not been implemented in FEniCS or Elmer/Ice for the current study, but will be included in a future study. The (linear) LPS method has also been extended to anisotropic meshes in [17].

\subsection{Sensitivity to stability parameter on the ISMIP-HOM domain}

For the ISMIP-HOM domain we use the same type of mesh resolution and cell size definition as in Section 5.1, i.e., $20 \times 20$ and $h_{K}=h_{\text {min }}$. As can be seen in Fig. 16, the relative errors for the LPS method is slightly more robust than the GLS method. The PP method shows a much narrower span for both the pressure and velocity, and results in high pressure errors for all $\tau_{0}$. Both the IP and PGP methods have a wide span and a low relative error for the vertical velocity, even for high values of $\tau_{0}=10^{3}-10^{4}$. PGP has the lowest relative errors for both pressure and velocity over the largest range of $\tau_{0}$. The PGP and IP methods both performed very well under even more extreme conditions, with $\tau_{0}=10^{4}$ giving relative errors in velocity of $10^{-1}$. We have not in this study thoroughly examined the possibility that the methods would be even more robust if the stabilization parameter were allowed to vary with the viscosity. However, initial simulations for the IP method indicate that this is not the case.

We also performed the horizontal mesh refinement experiment of Section 6.1.1 with all of the above stabilization methods. The results were qualitatively similar to the results obtained with GLS and both the PGP and IP methods performed well over a wide span of $\tau_{0}$.

\subsection{The outlet glacier}

The results from previous section indicate that the IP and PGP methods are accurate for a wide span of stabilization parameters. We therefore choose them to make further investigations, and here repeat the outlet glacier experiment from Section 5.2. The results of the first scenario with

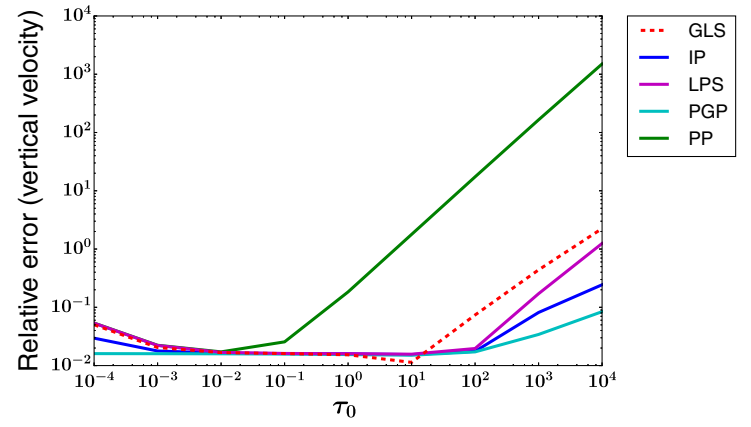

(a) Vertical velocity

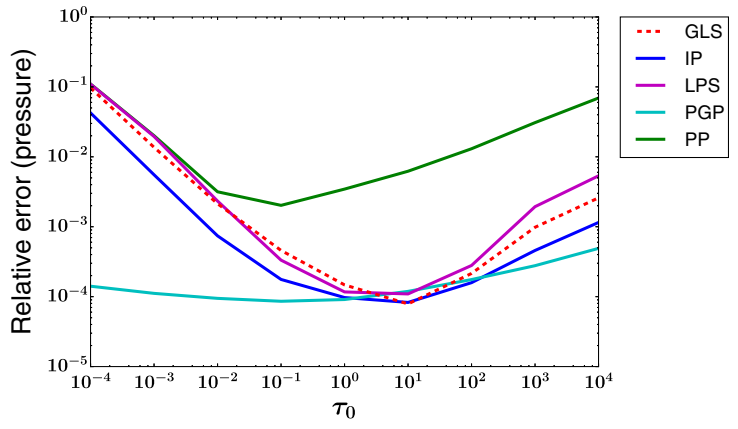

(b) Pressure

Fig. 16 The accuracy of alternative stabilization methods, and their sensitivity to the parameter $\tau_{0}$. The $L^{2}$-norm of the relative errors in vertical velocity and pressure are computed using a numerical reference solution 
frozen basal conditions and non-linear viscoity is shown in Fig. 17. The figure shows the changes in the vertical surface velocities when compared to an unstabilized, non-oscillatory, velocity field, for both IP and PGP, using both $h_{K}=$ $h_{\max }$ and $h_{K}=h_{\min }$. As before, the stabilization parameters are ranging four orders of magnitude; the smallest stability parameter shown is the smallest value needed to stabilize the pressure.

The range of possible stability parameters for these two methods are substantially narrowed compared to the ISMIPHOM case of Section 7.2, because of the inlet boundary condition. However, as for the ISMIP-HOM problem, the IP and the PGP methods are more robust than GLS with regards to the stabilization parameter. In the interior the effects of over-stabilization is much less severe than for the GLS method, and also the frontal oscillations are reduced. For the second scenario using basal sliding, the IP and PGP methods perform even better when compared to GLS method, and for the linear viscoity scenario, all oscillations are extremely small for both the PGP and IP method.

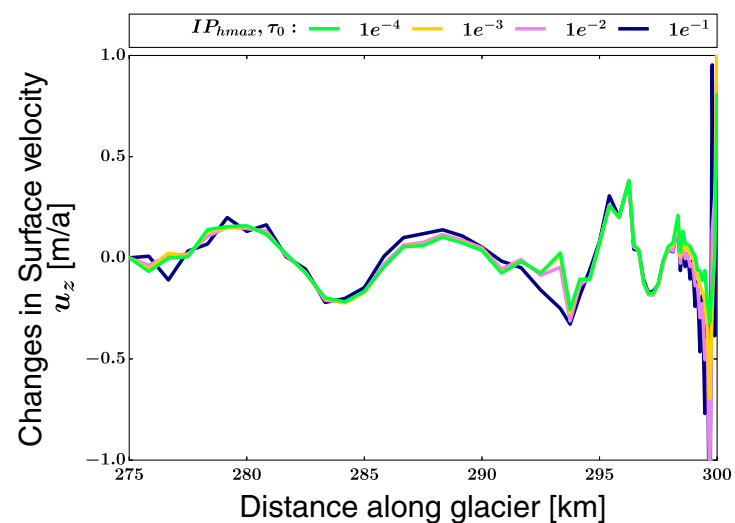

(a) IP, $h_{\max }$

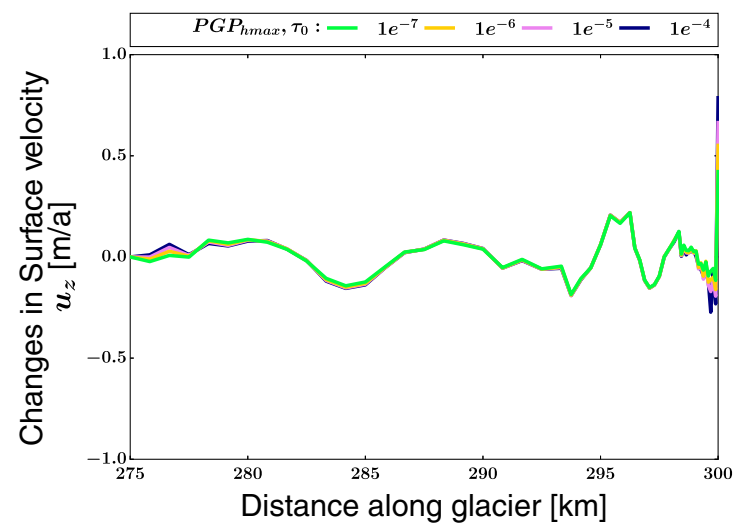

(c) PGP, $h_{\max }$

\subsection{Poiseuille flow}

As a final experiment, we examine how the IP method compares to the GLS method for the Poiseuille flow experiment. We choose the IP method due to its fairly low computational cost, which is comparable to the GLS method. This is a significant benefit compared to the PGP method. Similarly as for the GLS method, we here choose $h_{K}$ to be the maximum cell edge.

Compared to the GLS method in Fig. 12a, the curvature of the pressure contours in the upper left and lower right is not as evident in the IP method, see Fig. 18a. Like for the ISMIP-HOM problem and the outlet glacier case, the IP method is not prone to over-stabilization (Fig. 18b). The pressure artifacts due to the singularity is present also for the IP method, although they are much smaller and disappear for a high stabilization parameter, and for large values of $\tau_{0}$ the method converges to the analytical solution, even at the point of singularity (compare Fig. 12b and Fig. 18b).

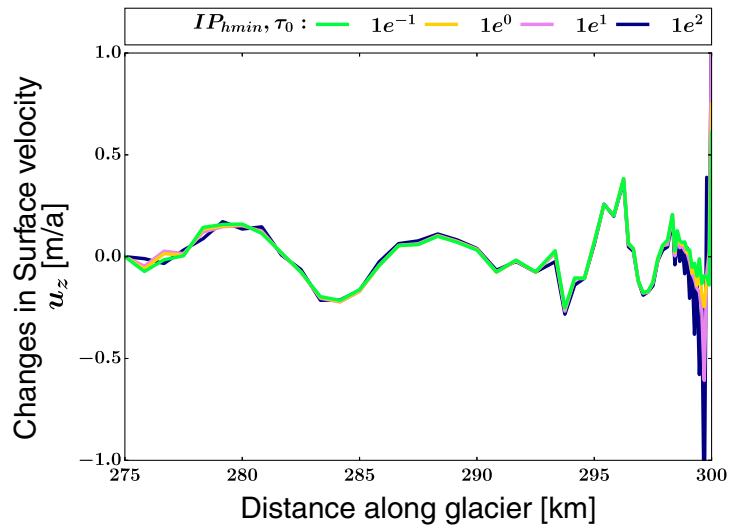

(b) IP, $h_{\min }$

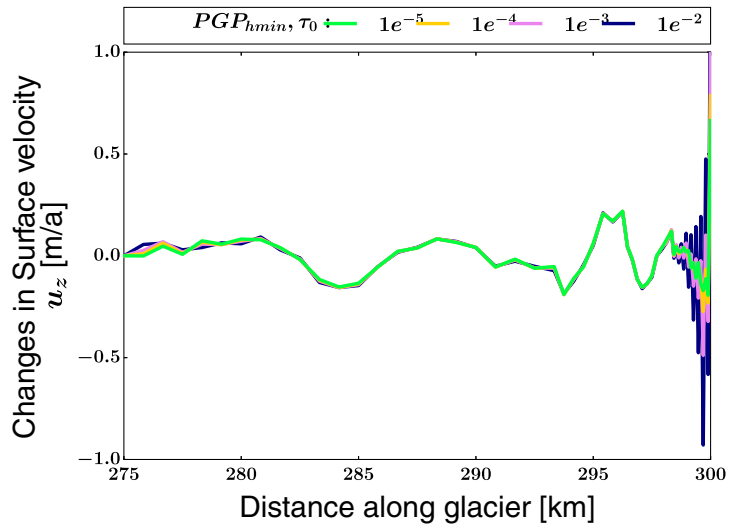

(d) PGP, $h_{\min }$

Fig. 17 Changes in vertical velocity for IP and PGP with different values of $\tau_{0}$, compared to an unstabilized velocity solution. The values of $\tau_{0}$ in each plot have been chosen such that any smaller values produced visible oscillations in the pressure field 


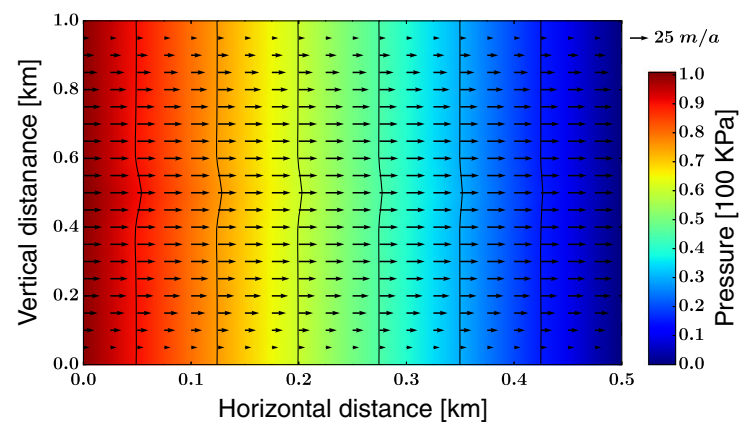

(a) IP method, $\tau_{0}=1$

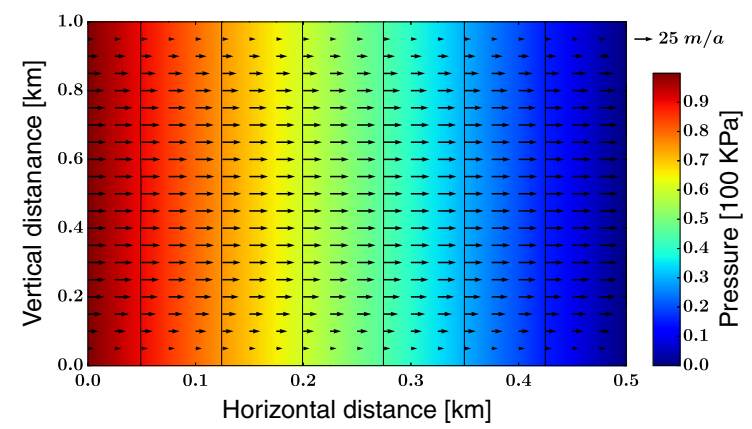

(b) IP method, $\tau_{0}=100$

Fig. 18 Poiseuille computed with IP stabilization. The stabilization parameter is specified with $\tau_{0}=1$ (left column) or $\tau_{0}=100$ (right column). Velocity is depicted as black arrows and pressure as color with black lines as pressure contours

\section{Summary and conclusions}

Stabilized low-order finite element methods have been extensively used in numerical ice-sheet modeling. However, the stabilization parameter is often chosen in an heuristic or ad hoc manner, without knowledge of the possible effects this choice can have on over- and under-stabilization of the problem. Choosing a sub-optimal value for the stabilization parameter can affect the accuracy of the solution, which can have a particularly negative effect when coupled to other numerical models of for instance the evolution of the free surface or the subglacial hydrological system.

We have here systematically investigated the accuracy and robustness of stabilized equal methods for the non-linear $\mathfrak{p}$-Stokes equations with application to ice-sheet modeling. We have done so keeping a common goal in glaciology in mind-accurate ice volume predictions. We focused on the commonly used GLS method, and compared it with other available stabilization techniques, namely a pressure penalty method, the interior penalty method, the pressure global projection method, and the local projection stabilization. We have mostly relied on numerical experiments, as the anisotropic meshes, non-linear rheology of ice and complex boundary conditions and geometries do not (yet) allow for analysis for a realistic set-up. We have mainly been working with the FEM code FEniCS ( $P 1 P 1$ element) in the numerical experiments, but to reduce the risk of erroneous implementations and measuring techniques, and to utilize some special functionalities, we have complemented some experiments with Elmer/Ice ( $Q 1 Q 1$ element).

We used both a numerical reference solution and the manufactured solutions presented in [45] to compute errors. We choose to follow earlier studies and measured the errors in the $L^{2}$ norm instead of the more natural $L^{\mathfrak{p} /(\mathfrak{p}-1)}$ and $W^{1, \mathfrak{p}}$ norm. When comparing with the numerical reference solution on the two-dimensional ISMIP-HOM B problem, a second-order convergence in both FEniCS and Elmer/Ice was observed. Using the manufactured solutions the convergence rate is two in FEniCS and three in Elmer/Ice. The surprising third-order convergence was found also in [32], regardless of using stabilized lower-order elements or unstabilized higher-order elements. We believe the explanation for this is high artificial surface forces at singularities in the manufactured problem. The manufactured problem is very sensitive to the implementation of the singular viscosity and artificial surface forces. This results in a super-convergence when refining the mesh in the vertical direction (but not in the horizontal plane) in Elmer/Ice. The strong artificial forces also results in a misrepresentation of the distribution of errors in the ice domain, and overestimates the error in the vertical velocity component. We therefore recommend great caution if using the manufactured solutions for evaluating the accuracy of ice-sheet models. We worked with the numerical reference solution in the remainder of the experiments of the paper, except for in one experiment.

The errors of the $\mathfrak{p}$-Stokes equations propagate into the calculations of the ice surface position. Due to the form of the ice surface evolution equation, the relevant errors to control are the errors in the vertical surface velocity component. These errors accumulate in time.

Unfortunately, since ice sheets are very thin, the vertical velocity is the variable that is the most sensitive to overstabilization, and it is thus important to choose an appropriate stabilization and stabilization parameter in order to accurately calculate the ice surface position. Over-stabilization may happen if the method as given by analysis is not appropriate for the specific problem, or if the variables of the parameter pre-multiplying the term in the GLS formulation is not defined appropriately. The classical stabilization parameter as suggested in [31] depends on the finite element cell size and the implementation of viscosity. If the cell size is chosen as the minimum edge length and the viscosity is allowed to vary non-linearly in the stabilization parameter, the value recommended in [31] is appropriate for the simple 
ISMIP-HOM problem. However, the optimal value of the stability parameter does depend on the element aspect ratio, and a horizontal mesh refinement may therefore not always give the expected gain in accuracy.

On a more realistic outlet glacier simulation with inlet velocity conditions, the value from [31] is unfortunately too large and results in velocity oscillations at the glacier front. This is true for both frozen basal conditions as well as sliding conditions. The oscillations are more severe because of the non-linear viscosity, and are amplified by both high viscosity gradients and low viscosity values. The GLS method also introduce artificial boundary conditions for ice flow. Hypothetically, this could cause inaccuracies in region with hydrodynamic pressure components, such as at the grounding line, but further investigations are needed to determine if this is an important issue in realistic simulations.

Out of the alternative stabilization methods tested, the simple pressure penalty was more sensitive to the stabilization parameter than GLS and was also less accurate. The (linear) local projection stabilization was slightly more robust compared to GLS in terms of sensitivity to the stability parameter and accuracy, but is more complicated to implement. It is possible that the non-linear version presented in [39] would improve its properties. Both the pressure global projection method and the interior penalty method showed very good stability results on the ISMIP-HOM problem. On the more realistic outlet glacier simulation the range of viable stability parameter values narrowed for these methods, although it was still wider than for the GLS method and the method was less prone to suffer from over-stabilization. The oscillations in IP and PGP seems to be almost entirely related to the non-linear viscosity, as they diminish for a linear viscosity. The difference in performance between GLS and IP or PGP was even more prominent if basal sliding was introduced. Due to its high computational cost, we do however not recommend the pressure global projection method for ice-sheet simulations, and the most attractive alternative method is thus the interior penalty method. This method was also less prone to introduce artificial boundary conditions and handle singularities in the viscosity.

In summary, standard equal order GLS-stabilized methods perform well on simplistic problems, but for more realistic glaciological problems inaccuracies in the vertical velocities may become large and affect the accuracy of the free surface position and thereby ice volume predictions. Care should therefore be taken in choosing the stability parameter as recommendations from literature may not automatically guarantee good results. A more robust alternative is the interior penalty method.

Acknowledgments Christian Helanow was supported by the nuclear waste management organizations in Sweden (Svensk Kärnbränslehantering $\mathrm{AB}$ ) and Finland (Posiva $\mathrm{Oy}$ ) through the Greenland Analogue Project and by Gålöstiftelsen. Josefin Ahlkrona was supported by the
Swedish strategic research programme eSSENCE (Uppsala) and the cluster of Excellence 80 "The Future Ocean" (Kiel). The "Future Ocean" is funded within the framework of the Excellence Initiative by the Deutsche Forschungsgemeinschaft (DFG) on behalf of the German federal and state governments. The computations with Elmer/Ice were performed on resources provided by the Swedish National Infrastructure for Computing (SNIC) at PDC Centre for High Performance Computing (PDC-HPC) and at Uppmax at Uppsala University. Both facilities provided excellent support. We wish to thank two anonymous reviewers for their constructive criticism, and Per Lötstedt and Peter Jansson for valuable comments on the manuscript.

Open Access This article is distributed under the terms of the Creative Commons Attribution 4.0 International License (http:// creativecommons.org/licenses/by/4.0/), which permits unrestricted use, distribution, and reproduction in any medium, provided you give appropriate credit to the original author(s) and the source, provide a link to the Creative Commons license, and indicate if changes were made.

\section{References}

1. Ahlkrona, J., Kirchner, N., Lötstedt, P.: Accuracy of the zeroth and second order shallow ice approximation - numerical and theoretical results. Geosci. Model Dev. 6, 2135-2152 (2013)

2. Ainsworth, M., Coggins, P.: The stability of mixed hp-finite element methods for stokes flow on high aspect ratio elements. SIAM J. Numer. Anal. 38(5), 1721-1761 (2000)

3. Alley, R.B., MacAyeal, D.R.: Ice-rafted debris associated with binge/purge oscillations of the Laurentide ice sheet. Paleoceanography 9(4), 503-511 (1994)

4. Alnæs, M., Blechta, J., Hake, J., Johansson, A., Kehlet, B., Logg, A., Richardson, C., Ring, J., Rognes, M., Wells, G.: The FEniCS Project Version 1.5 Archive of Numerical Software 3(100) (2015)

5. Alnæs, M.S., Logg, A., Olgaard, K.B., Rognes, M.E., Wells, G.N.: Unified form language: a domain-specific language for weak formulations of partial differential equations. ACM Trans. Math. Softw. 40(2), 9:1-9:37 (2014)

6. Arnold, D.N., Brezzi, F., Fortin, M.: A stable finite element for the Stokes equations. CALCOLO 21(4), 337-344 (1984)

7. Babuška, I.: The finite element method with Lagrangian multipliers. Numer. Math. 20(3), 179-192 (1973)

8. Baiocchi, C., Brezzi, F., Franca, L.P.: Virtual bubbles and Galerkin-least-squares type methods (ga.l.s.) Comput. Methods Appl. Mech. Eng. 105(1), 125-141 (1993)

9. Balay, S., Abhyankar, S., Adams, M.F., Brown, J., Brune, P., Buschelman, K., Dalcin, L., Eijkhout, V., Gropp, W.D., Kaushik, D., Knepley, M.G., McInnes, L.C., Rupp, K., Smith, B.F., Zampini, S., Zhang, H., Zhang, H.: PETSC Web page http://www. mcs.anl.gov/petsc (2016)

10. Bamber, J.: Greenland $5 \mathrm{~km}$ DEM, ice thickness, and bedrock elevation grids https://nsidc.org/data/docs/daac/nsidc0092_greenland_ ice_thickness.gd.html (2001)

11. Becker, R., Braack, M.: A finite element pressure gradient stabilization for the Stokes equations based on local projections. CALCOLO 38(4), 173-199 (2001)

12. Belenki, L., Berselli, L.C., Diening, L., Růžička, M.: On the finite element approximation of p-stokes systems. SIAM J. Numer. Anal. 50(2), 373-397 (2012)

13. Bindschadler, R.A., Nowicki, S., Abe-Ouchi, A., Aschwanden, A., Choi, H., Fastook, J., Granzow, G., Greve, R., Gutowski, G., Herzfeld, U., Jackson, C., Johnson, J., Khroulev, C., Levermann, A., Lipscomb, W.H., Martin, M.A., Morlighem, M., Parizek, B.R., Pollard, D., Price, S.F., Ren, D., Saito, F., Sato, T., Seddik, H., 
Seroussi, H., Takahashi, K., Walker, R., Wang, W.L.: Ice-sheet model sensitivities to environmental forcing and their use in projecting future sea level (the seaRISE project). J. Glaciol. 59, 195-224 (2013). http://tinyurl.com/srise-umt

14. Blasco, J.: An anisotropic GLS-stabilized finite element method for incompressible flow problems. Comput. Methods Appl. Mech. Eng. 197(45-48), 3712-3723 (2008)

15. Blatter, H.: Velocity and stress fields in grounded glaciers: a simple algorithm for including deviatoric stress gradients. J. Glaciol. 41, 333-344 (1995)

16. Bochev, P.B., Dohrmann, C.R., Gunzburger, M.: Stabilization of mixed finite elements for the stokes equations. SIAM J. Numerical Analysis 44, 82-101 (2006)

17. Braack, M.: A stabilized finite element scheme for the navierstokes equations on quadrilateral anisotropic meshes. ESAIM: Mathematical Modelling and Numerical Analysis 42(6), 903-924 (2008)

18. Braack, M., Burman, E., John, V., Lube, G.: Stabilized finite element methods for the generalized oseen problem. Comput. Methods Appl. Mech. Engrg. 196, 853-866 (2007)

19. Braack, M., Lube, G.: Finite elements with local projection stabilization for incompressible flow problems. J. Comput. Math. 27(2/3), 116-147 (2009)

20. Brezzi, F.: On the existence, uniqueness and approximation of saddle-point problems arising from lagrangian multipliers. ESAIM: Mathematical Modelling and Numerical Analysis Modé,lisation Mathématique et Analyse Numérique 8(R2), 129151 (1974)

21. Brezzi, F., Fortin, M.: Mixed and Hybrid Finite Element Methods. Springer, New York (1991)

22. Brinkerhoff, D.J., Johnson, J.V.: Data assimilation and prognostic whole ice sheet modelling with the variationally derived, higher order, open source, and fully parallel ice sheet model varGlaS. Cryosphere 7, 1161-1184 (2013)

23. Burman, E., Hansbo, P.: Edge stabilization for the generalized stokes problem: a continuous interior penalty method. Comput. Methods Appl. Mech. Eng. 195(19-22), 2393-2410 (2006)

24. Calov, R., Marsiat, I.: Simulations of the Northern hemisphere through the last glacial-interglacial cycle with a vertically integrated and a three-dimensional thermomechanical ice-sheet model coupled to a climate model. Ann. Glaciol. 27, 169-176 (1998)

25. Chen, Q., Gunzburger, M., Perego, M.: Well-posedness results for a nonlinear stokes problem arising in glaciology. SIAM J. Math. Anal. 45(5), 2710-2733 (2013)

26. Church, J., Clark, P., Cazenave, A., Gregory, J., Jevrejeva, S., Levermann, A., Merrifield, M., Milne, G., Nerem, R., Nunn, P., Payne, A., Pfeffer, W., Stammer, D., Unnikrishnan, A.: Sea level change. In: Stocker, T., Qin, D., Plattner, G.K., Tignor, M., Allen, S., Boschung, J., Nauels, A., Xia, Y., Bex, V., Midgley, P. (eds.) Climate Change 2013: the Physical Science Basis. Contribution of Working Group I to the Fifth Assessment Report of the Intergovernmental Panel on Climate Change, Book Section 13, pp. 1137-1216. Cambridge University Press, Cambridge (2013)

27. Codina, R., Blasco, J.: A finite element formulation for the Stokes problem allowing equal velocity-pressure interpolation. Comput. Methods Appl. Mech. Eng. 143(3), 373-391 (1997)

28. Davis, T.A.: SuiteSparse Web page http://faculty.cse.tamu.edu/ davis/suitesparse.html (2016)

29. Droux, J.J., Hughes, T.J.: A boundary integral modification of the Galerkin least squares formulation for the Stokes problem. Comput. Methods Appl. Mech. Eng. 113(1), 173-182 (1994)

30. Ern, A., Guermond, J.L.: Theory and Practice of Finite Elements. Applied Mathematical Sciences. Springer, New York (2004)

31. Franca, L., Frey, S.: Stabilized finite element methods: II. The incompressible navier-stokes equations. Comput. Methods Appl. Mech. Eng. 99, 209-233 (1992)
32. Gagliardini, O., Zwinger, T., Gillet-Chaulet, F., Durand, G., Favier, L., de Fleurian, B., Greve, R., Malinen, M., Martín, C., Råback, P., Ruokolainen, J., Sacchettini, M., Schäfer, M., Seddik, H., Thies, J.: Capabilities and performance of Elmer/Ice, a new generation ice-sheet model. Geosci. Model Dev. 6, 1299-1318 (2013)

33. Gillet-Chaulet, F., Gagliardini, O., Seddik, H., Nodet, M., Durand, G., Ritz, C., Zwinger, T., Greve, R., Vaughan, D.G.: Greenland ice sheet contribution to sea-level rise from a new-generation ice-sheet model. Cryosphere 6, 1561-1576 (2012)

34. Gmeiner, B., Rüde, U., Stengel, H., Waluga, C., Wohlmuth, B.: Performance and scalability of hierarchical hybrid multigrid solvers for stokes systems. SIAM J. Sci. Comput. 37(2), C143C168 (2015)

35. Greve, R.: Thermomechanisches Verhalten Polythermer Eisschilde - Theorie, Analytik, Numerik. Ph.D. Thesis Department of Mechanics, vol. III. Technical University Darmstadt, Germany (1995)

36. Greve, R.: A continuum-mechanical formulation for shallow polythermal ice sheets. Phil. Trans. R. Soc. Lond. A 355, 921-974 (1997)

37. Heinrich, H.: Origin and consequences of cyclic ice rafting in the Northeast Atlantic Ocean during the past 130,000 years. Quatern. Res. 29(2), 142-152 (1988)

38. Hirn, A.: Finite Element Approximation of Problems in NonNewtonian Fluid Mechanics. Ph.D. thesis, Mathematical Institute of Charles University (2011)

39. Hirn, A.: Approximation of the p-stokes equations with equalorder finite elements. J. Math. Fluid Mech. 15(1), 65-88 (2012)

40. Hughes, T.J.R., Franca, L.P., Balestra, M.: A new finite element formulation for computational fluid dynamics: V. Circumventing the Babuška-Brezzi Condition: a stable PetrovGalerkin formulation of the stokes problem accommodating equal-order interpolations. Comput. Methods Appl. Mech. Eng. 59(1), 85-99 (1986)

41. Huybrechts, P.: A 3-D model for the Antarctic ice sheet: a sensitivity study on the glacial-interglacial contrast. Climate Dynam. 5(2), 79-92 (1990)

42. John, V., Knobloch, P., Novo, J.: Finite elements for scalar convection-dominated equations and incompressible flow problems - A never ending story? Weierstrass Institute for Applied Analysis and Stochastics: Preprint 2410. http://www.wias-berlin. de/preprint/2410/wias_preprints_2410.pdf. Preprint (2017)

43. Jouvet, G., Rappaz, J.: Analysis and finite element approximation of a nonlinear stationary stokes problem arising in glaciology advances in numerical analysis (2011)

44. Larour, E., Seroussi, H., Morlighem, M., Rignot, E.: Continental scale, high order, high spatial resolution, ice sheet modeling using the Ice Sheet System Model (ISSM). J. Geophys. Res. 117, F01,022 (2012)

45. Leng, W., Ju, L., Gunzburger, M., Price, S.: Manufactured solutions and the verification of three-dimensional stokes icesheet models. Cryosphere 7, 19-29 (2013)

46. Leng, W., Ju, L., Xie, Y., Cui, T., Gunzburger, M.: Finite element three-dimensional stokes ice sheet dynamics model with enhanced local mass conservation. J. Comput. Phys. 274, 299-311 (2014)

47. Logg, A., Mardal, K.A., Wells, G.N.: Automated Solution of Differential Equations by the Finite Element Method, Lecture Notes in Computational Science and Engineering, vol. 84. Springer, Berlin (2012)

48. Micheletti, S., Perotto, S., Picasso, M.: Stabilized finite elements on anisotropic meshes: a priori error estimates for the advectiondiffusion and the stokes problems. SIAM J. Numer. Anal. 41(3), 1131-1162 (2003)

49. Pattyn, F.: A new three-dimensional higher-order thermomechanical ice sheet model: basic sensitivity, ice stream development, 
and ice flow across subglacial lakes. J. Geophys. Res. 108, 2382 (2003)

50. Pattyn, F., Perichon, L., Aschwanden, A., Breuer, B., de Smedt, B., Gagliardini, O., Gudmundsson, G.H., Hindmarsh, R., Hubbard, A., Johnson, J.V., Kleiner, T., Konovalov, Y., Martin, C., Payne, A.J., Pollard, D., Price, S., Rückamp, M., Saito, F., Souček, O., Sugiyama, S., Zwinger, T.: Benchmark experiments for higher-order and full-Stokes ice sheet models (ISMIP-HOM). Cryosphere 2, 95-108 (2008)

51. Petra, N., Zhu, H., Stadler, G., Hughes, T.J.R., Ghattas, O.: An inexact gauss-Newton method for inversion of basal sliding and rheology parameters in a nonlinear Stokes ice sheet model. J. Glaciol. 58, 889-903 (2012)

52. Råback, P., Malinen, M., Ruokalainen, J., Pursula, A., Zwinger, T.: Elmer Models Manual. CSC - IT Center for Science, Helsinki (2013)

53. Sargent, A., Fastook, J.L.: Manufactured analytical solutions for isothermal full-stokes ice sheet models. Cryosphere 4(3), 285-311 (2010)

54. Seddik, H., Greve, R., Zwinger, T., Gillet-Chaulet, F., Gagliardini, O.: Simulations of the Greenland ice sheet 100 years into the future with the full Stokes model elmer/Ice. J. Glaciol. 58, 427440 (2012)
55. Shewchuk, J.R.: What is a good linear finite element? - interpolation, conditioning, anisotropy, and quality measures. Tech. rep. In: Proceedings of the 11th international meshing roundtable (2002)

56. Taylor, C., Hood, P.: Navier-stokes equations using mixed interpolation. In: International symposium on finite element methods in flow problems, pp. 121-132 (1974)

57. Apel, T., Knopp, T., Lube, G.: Stabilized finite element methods with anisotropic mesh refinement for the oseen problem. Appl. Numer. Math. 58, 1830-1843 (2008)

58. Turner, D.Z., Nakshatrala, K.B., Hjelmstad, K.D.: On the stability of bubble functions and a stabilized mixed finite element formulation for the stokes problem. Int. J. Numer. Methods Fluids 60(12), 1291-1314 (2009)

59. Vialov, S.: Regularities of glacial shields movement and the theory of plastic viscous flow. IAHS 47, 266-275 (1958)

60. Zhang, H., Ju, L., Gunzburger, M., Ringler, T., Price, S.: Coupled models and parallel simulations for three-dimensional full-Stokes ice sheet modeling. Numer. Math. Theor. Meth. Appl. 4, 359-381 (2011)

61. Zwinger, T., Greve, R., Gagliardini, O., Shiraiwa, T., Lyly, M.: A full Stokes-flow thermo-mechanical model for firn and ice applied to the Gorshkov crater glacier. Kamchatka Ann. Glaciol. 45(1), 29-37 (2007) 